\title{
Collapsing Geometry with Ricci Curvature Bounded Below and Ricci Flow Smoothing
}

Shaosai $H U A N G^{\dagger}$, Xiaochun RONG $\ddagger$ and Bing $W A N G \S$

$\dagger$ Department of Mathematics, University of Wisconsin-Madison, Madison, WI 53706, USA

E-mail: sshuang@math.wisc.edu

URL: https://sites.google.com/a/wisc.edu/shaosai-huang

$\ddagger$ Department of Mathematics, Rutgers University, New Brunswick, NJ 08854, USA

E-mail: rong@math.rutgers.edu

$\S$ Institute of Geometry and Physics, and School of Mathematical Sciences, University of Science and Technology of China, Hefei, Anhui Province, 230026, China

E-mail: topspin@ustc.edu.cn

URL: http://staff.ustc.edu.cn/ topspin/index.html

Received August 30, 2020, in final form November 23, 2020; Published online November 30, 2020 https://doi.org/10.3842/SIGMA.2020.123

\begin{abstract}
We survey some recent developments in the study of collapsing Riemannian manifolds with Ricci curvature bounded below, especially the locally bounded Ricci covering geometry and the Ricci flow smoothing techniques. We then prove that if a Calabi-Yau manifold is sufficiently volume collapsed with bounded diameter and sectional curvature, then it admits a Ricci-flat Kähler metric together with a compatible pure nilpotent Killing structure: this is related to an open question of Cheeger, Fukaya and Gromov.
\end{abstract}

Key words: almost flat manifold; collapsing geometry; locally bounded Ricci covering geometry; nilpotent Killing structure; Ricci flow

2020 Mathematics Subject Classification: 53C21; 53C23; 53E20

\section{Introduction}

In the seminal work [39], Gromov discovered a gap phenomenon for the sectional curvature (denoted by $\mathbf{K}_{g}$ for a smooth Riemannian metric $g$ ) to detect the infranil manifold structure (see also $[4,79])$ :

Theorem 1.1 (Gromov's almost flat manifold theorem, 1978). There is a dimensional constant $\varepsilon(m) \in(0,1)$ such that if a closed $m$-dimensional Riemannian manifold $(M, g)$ satisfies

$$
\operatorname{diam}(M, g)^{2} \max _{\wedge^{2} T M}\left|\mathbf{K}_{g}\right| \leq \varepsilon^{2},
$$

then $M$ is diffeomorphic to an infranil manifold.

Here we say that $M$ is an infranil manifold if on the universal covering $\tilde{M}$ of $M$ there is a flat connection with parallel torsion, defining a simply connected nilpotent Lie group structure $N$ on $\tilde{M}$ such that $\pi_{1}(M)$ is a sub-group of $N \rtimes \operatorname{Aut}(N)$ with $\left[\pi_{1}(M): \pi_{1}(M) \cap N\right]<\infty$ and $\operatorname{rank} \pi_{1}(M)=m$ - in the case of Gromov's almost flat manifold theorem, it is also shown that such index has a uniform dimensional upper bound $C(m)$.

This paper is a contribution to the Special Issue on Scalar and Ricci Curvature in honor of Misha Gromov on his 75th Birthday. The full collection is available at https://www.emis.de/journals/SIGMA/Gromov.html 
Ever since its birth, Gromov's almost flat manifold theorem has inspired the research of Riemannian geometers by two themes of generalizations. One theme is to find parametrized versions of Theorem 1.1, as indicated by Fukaya's fiber bundle theorem [34]: if a Riemannian manifold with bounded diameter and sectional curvature is sufficiently Gromov-Hausdorff close to a lower dimensional one with bounded geometry, then it is diffeomorphic to the total space of a smooth family of almost flat manifolds parametrized over the lower dimensional one (see Theorem 2.1). The generalized version of this theorem (see Theorem 2.2), combined with the more intrinsic approach of Cheeger and Gromov [16, 17] on $F$-structures of positive rank, whose existence is equivalent to the existence of a one-parameter family of Riemannian metrics collapsing with bounded sectional curvature, nurtured the rich and splendent theory of the collapsing geometry with bounded sectional curvature, notably the construction (in [14]) and various applications of the nilpotent Killing structure (see Theorem 2.3). This theory, as we will briefly recall in Section 2.1, is mainly developed through the works of Cheeger and Gromov [16, 17], Fukaya [33, 34, 35, 36], and Cheeger, Fukaya and Gromov [14].

The other theme of generalization focuses on weakening the curvature assumption (1.1) to lower (Bakry-Émery) Ricci curvature bounds, as examplified by the Colding-Gromov gap theorem: if a Ricci almost non-negatively curved manifold of unit diameter has its first Betti number equal to its dimension, then the manifold is diffeomorphic to a flat torus (see Theorem 2.5). Obviously, the weaker curvature assumption alone is insufficient to conclude the infranil manifold structure, and certain extra assumptions are necessary - just as the case of the Colding-Gromov gap theorem; see also [30, 49, 55, 69] and Section 2.2 for a brief overview.

The two streams of research become confluent in the study of collapsing Riemannian manifolds with Ricci curvature bounded below. The exploration in this field is driven not just by its intrinsic merit of finding weaker curvature assumptions, but also externally by the study of Kähler geometry and mathematical physics: a major open problem in string theory is the SYZ conjecture [81], which could be formulated as understanding the collapsing geometry of Ricci-flat Kähler metrics: see, e.g., [58, 60, 83]; see also [41, 42, 43, 46] for some examples concerning such phenomena.

The weaker curvature assumption causes tremendous difficulties for our understanding, and it is natural to start with some extra assumptions. For instance, one could impose some extra conditions on topology such as the first Betti numbers: in [53], it is shown by the first- and thirdnamed authors that if a Riemannian manifold with Ricci curvature bounded below is sufficiently Gromov-Hausdorff close to a lower dimensional one with bounded geometry, and the difference of their first Betti numbers is equal to their dimensional difference, then the higher dimensional manifold is diffeomorphic to a torus bundle over the lower dimensional one (see Theorem 2.11). This theorem generalizes Fukaya's fiber bundle theorem and the Colding-Gromov gap theorem simultaneously, in setting of collapsing Riemannian manifolds with Ricci curvature bounded below - related results will be surveyed in Section 2.3.

Since the collapsing phenomenon with bounded sectional curvature is essentially due to the abundant local symmetry encoded in the local fundamental group, a very natural condition is to assume that the local universal covering space around every point is non-collapsing: this is the program initiated by the second-named author and his collaborators in [49] to systematically investigate those manifolds with locally bounded Ricci covering geometry, which we will discuss in Section 3. In fact, the major effort in proving Theorem 2.11 is devoted to decoding the topological information associated with the first Betti numbers and show that the higher dimensional manifold has locally bounded Ricci covering geometry.

A key issue in the study of collapsing Riemannian manifolds with Ricci curvature bounded below is the low regularity of the metric due to the (weaker) Ricci curvature assumption. This confines our understanding on the finer structures of the collapsing geometry, and suitable smoothing of the given metric is usually inevitable - in Section 4, we will survey the relevant 
Ricci flow smoothing techniques for locally collapsing manifolds with Ricci curvature bounded below.

In fact, the Ricci flow smoothing technique also enhances our understanding on the classical theory of collapsing with bounded sectional curvature. Cheeger, Fukaya and Gromov asked in [14] the following question which remains open today.

Question 1.2. It is known that given a complete Riemannian manifold $(M, g)$ with sectional curvature uniformly bounded between \pm 1 , for any small $\varepsilon>0$ there is a regular $(\rho, k)$-round metric $g_{\varepsilon}$ and nilpotent Killing structure $\mathfrak{N}$ compatible with $g_{\varepsilon}$, such that $\left\|g-g_{\varepsilon}\right\|_{C^{1}}<\varepsilon$. Now if the initial metric $g$ is assumed to be Kähler or Einstein, can we find $g_{\varepsilon}$ in the same category?

In the last section of this note, we will prove, based on Ricci flow techniques, that if a Ricciflat Kähler metric is very collapsed with bounded diameter and sectional curvature, then the approximating metric compatible with a nilpotent Killing structure may indeed be found as a nearby Ricci-flat Kähler metric. Notice that the approximating metric $g_{\varepsilon}$ obtained from [14, Theorem 1.7] is not necessarily Kähler or Ricci-flat, but we manage to evolve it along the Ricci flow to find a desirable one. Since the Ricci flow respects local isometries, the evolved metrics are compatible with the original nilpotent Killing structure $\mathfrak{N}$. We wish our result could cast some light on the general case of Cheeger, Fukaya and Gromov's open question.

\section{Collapsing geometry with sectional or Ricci curvature bounds}

In this section we give a short survey of the two directions generalizing Theorem 1.1 - the collapsing geometry with bounded sectional curvature and the almost flatness characterized by weaker curvature conditions - as well as the study of collapsing geometry with only Ricci curvature bounded below. While there have been comprehensive surveys on the theory of collapsing geometry [37, 75], we still briefly go through some classical theorems so as to put the study of collapsing geometry with Ricci curvature bounded below in the historical context.

\subsection{Collapsing with bounded sectional curvature}

Gromov's almost flat manifold theorem, when embedded in the framework of the coarse geometry on the space of all Riemannian manifolds (see [40]), opened a new chapter in the study of Riemannian geometry: the collapsing geometry of Riemannian manifolds with bounded curvature. In this note, we consider the following collections of Riemannian manifolds:

1) $\mathcal{M}_{\mathrm{Rm}}(m, D)$ denoting the collection of $m$-dimensional Riemannian manifolds with sectional curvature bounded between \pm 1 , and diameter bounded from above by $D \geq 1$;

2) $\mathcal{M}_{\mathrm{Rm}}(m, D, v)$ denoting the sub-collection of $\mathcal{M}_{\mathrm{Rm}}(m, D)$ with volume bounded below by $v>0$.

Equipped with the Gromov-Hausdorff topology, the moduli space $\mathcal{M}_{\mathrm{Rm}}(m, D)$ is pre-compact [40]. Based on the work of Cheeger (see [12,38]), the sub-collection $\mathcal{M}_{\operatorname{Rm}}(m, D, v)$ is not just compact in the Gromov-Hausdorff topology, but also has only finitely many diffeomorphism classes. On the contrary, if we consider a sequence $\left\{\left(M_{i}, g_{i}\right)\right\}$ in $\mathcal{M}_{\mathrm{Rm}}(m, D)$, then under the Gromov-Hausdorff topology, it is possible that $M_{i} \stackrel{\mathrm{GH}}{\longrightarrow} N$, for some lower dimensional manifold $(N, h)$ in $\mathcal{M}_{\mathrm{Rm}}(k, D, v)$ with $k<m$. In this case, we say that $\left\{\left(M_{i}, g_{i}\right)\right\}$ collapses to $N$ with bounded curvature and diameter. In [34] it is shown that such situation can only occur when $M_{i}$ are infranil fiber bundles over $N$.

Theorem 2.1 (Fukaya's fiber bundle theorem, 1987). Given $D \geq 1$ and $v>0$, there is a uniform constant $\varepsilon(m, v) \in(0,1)$ such that if $(M, g) \in \mathcal{M}_{\mathrm{Rm}}(m, D)$ and $(N, h) \in \mathcal{M}_{\mathrm{Rm}}(k, D, v)$ with 
$k \leq m$ satisfy $d_{\mathrm{GH}}(M, N)<\delta$ for some $\delta<\varepsilon(m, v)$, then there is a $C^{1}$ submersion $f: M \rightarrow N$ such that

1) $f$ is an almost Riemannian submersion, i.e., $\mathrm{e}^{-\Psi_{F}(\delta \mid m, v)}|\xi|_{g} \leq\left|f_{*} \xi\right|_{h} \leq \mathrm{e}^{\Psi_{F}(\delta \mid m, v)}|\xi|_{g}$ for any $\xi \perp \operatorname{ker} f_{*}$, with $\Psi_{F}(\delta \mid m, v) \in(0,1)$ satisfying $\lim _{\delta \rightarrow \infty} \Psi_{F}(\delta \mid m, v)=0$; and

2) the fiber of $f$ is diffeomorphic to an infranil manifold.

This theorem describes the diffeomorphism type of those sufficiently collapsed manifolds in $\mathcal{M}_{\mathrm{Rm}}(m, D)$ by those "minimal models" in $\mathcal{M}_{\mathrm{Rm}}(k, D, v)$, as long as we can find such a lower dimensional model space. In general however, we cannot expect a sequence in $\mathcal{M}_{\mathrm{Rm}}(m, D)$ to collapse to an element in $\mathcal{M}_{\mathrm{Rm}}(k, D, v)$. We will refer to those Hausdorff $k$-dimensional $(k<m)$ metric spaces arising as the Gromov-Hausdorff limits of sequences in $\mathcal{M}_{\mathrm{Rm}}(m, D)$ as the collapsing limit spaces. The local structure of such spaces is described in [35, Theorem 0.5]: for any point $x$ in a collapsing limit space $\left(X, d_{X}\right)$, there is an open neighborhood $V$ of $x$, a Lie group $G_{x}$ admitting a faithful representation to $\mathrm{O}(n)$ (for some $n \leq m$ ), and a $G_{x}$-invariant Riemannian metric $\tilde{g}$ on an open neighborhood $U$ of $\vec{o} \in \mathbb{R}^{n}$, such that the identity component of $G_{x}$ is isomorphic to a torus and $\left(V, d_{X}\right) \equiv(U, \tilde{g}) / G_{x}$. It is consequently shown in $[14,35,36]$ that a manifold $M \in \mathcal{M}_{\mathrm{Rm}}(m, D)$ sufficiently Gromov-Hausdorff close to a collapsing limit space $X$ exhibits a singular fibration over $X$ :

Theorem 2.2 (singular fibration, 1988-1992). Given $D \geq 1$, there are uniform constants $\varepsilon(m, D)$ and $c(m)>0$ to the following effect: if $(M, g) \in \mathcal{M}_{\mathrm{Rm}}(m, D)$ satisfies $|M|_{g}<\varepsilon \leq$ $\varepsilon(m, D)$, then the frame bundle $F(M)$ of $M$, equipped with the canonical metric $\bar{g}$, is GromovHausdorff close to some $(Y, h) \in \mathcal{M}_{\mathrm{Rm}}\left(m^{\prime}, D^{\prime}, v^{\prime}\right)$ with $m^{\prime}<m+\frac{1}{2} m(m-1)$ and $D^{\prime}, v^{\prime}>0$ determined by $m$ and $D$, such that $\mathrm{O}(m)$ acts isometrically on $(Y, h)$ and there is an $\mathrm{O}(m)$-equivariant fiber bundle $\bar{f}:(F(M), \mathrm{O}(m)) \rightarrow(Y, \mathrm{O}(m))$; the fiber of $\bar{f}$ is diffeomorphic to a compact nilmanifold $N / \Gamma$ (with $N$ being a simply connected nilpotent Lie group and $\Gamma \leq N$ a co-compact lattice), and the structure group is contained in $(C(N) /(C(N) \cap \Gamma)) \rtimes \operatorname{Aut}(\Gamma)$; moreover, $\bar{f}$ induces a singular fibration $f: M \rightarrow X=Y / \mathrm{O}(m)$ that fits into the following commutative diagram:

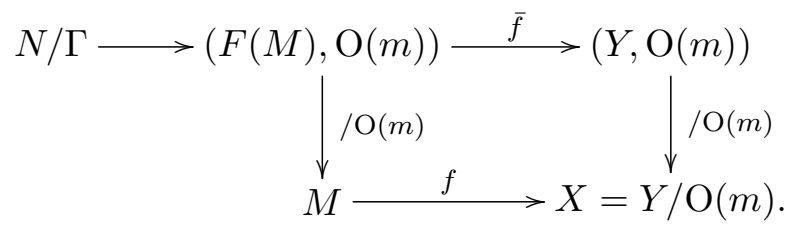

Finally, $\bar{f}$ is an $\Psi(\varepsilon \mid m, D)$-Gromov-Hausdorff approximation satisfying item (1) of Theorem 2.1, and the second fundamental form of each $\bar{f}$ fiber is uniformly bounded by $c(m)$ in magnitude.

Here the key observation is that two isometries of a Riemannian manifold are identical if their 1 -jets agree at some point, and the total space of 1 -jets of isometries is conveniently represented by self-maps of the frame bundle; see [35]. Notice that the above mentioned fiber bundle theorems are for manifolds collapsing also with bounded diameter, and they are in the differentiable category. However, due to the existence of abundant local symmetries for those very collapsed manifolds with bounded sectional curvature, it is natural to wonder if the extra symmetry provided by the infranil fibers can be reflected on the level of Riemannian metrics, locally around a given fiber. This direction has been studied by Cheeger and Gromov [16, 17] for the central part of the infranil fibers (constructing the $F$-structure), and is thoroughly investigated in the foundational work of Cheeger, Fukaya and Gromov [14]: on the very collapsed part of a complete Riemannian manifold with bounded sectional curvature, a nilpotent Killing structure of positive rank is constructed, providing the finest description of the collapsing geometry.

We now define the nilpotent structure (a.k.a. $N$-structure) on a given complete Riemannian manifold $(M, g)$. Roughly speaking, it is the local singular fiber bundle as in Theorem 2.2 patched 
together. Let $\left\{U_{j}\right\}$ be a locally finite open covering of $M$, then for each $U_{j}$ we can associate an elementary $N$-structure $\mathfrak{N}_{j}$, which is nothing but a singular fiber bundle $f_{j}: U_{j} \rightarrow X_{j}$ satisfying the description in Theorem 2.2 - this can be seen as a localization of that theorem, and all the information is encoded in the following commutative diagram

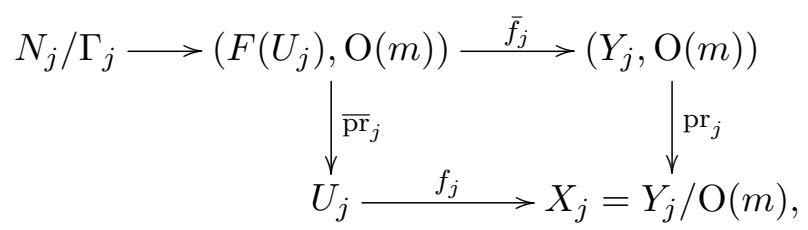

where $\overline{\mathrm{pr}}_{j}$ and $\mathrm{pr}_{j}$ denote the natural projections onto the space of $\mathrm{O}(m)$ orbits. By the commutativity of this diagram, we see for any $x \in X_{j}$ that $f_{j}^{-1}(x)=\overline{\operatorname{pr}}_{j}\left(\bar{f}_{j}^{-1}\left(\operatorname{pr}_{j}^{-1}(x)\right)\right)$ is an infranil manifold, called the $\mathfrak{N}_{j}$-orbit passing through any point of $f_{j}^{-1}(x)$; we let $\mathcal{O}_{j}(p)$ denote such an orbit passing through a given $p \in f_{j}^{-1}(x)$. An open set $V \subset U_{j}$ is said to be $\mathfrak{N}_{j}$-invariant if it is the union of $\mathfrak{N}_{j}$-orbits of points in $V$. An $N$-structure $\mathfrak{N}$ on $M$ is then a collection of elementary $N$-structure $\left\{\mathfrak{N}_{j}\right\}$ satisfying the compatibility condition: there is an ordering of $\{j\}$

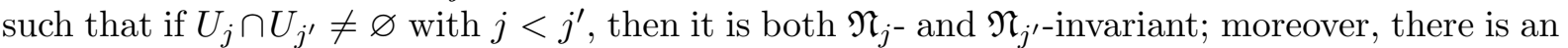
$\mathrm{O}(m)$-equivariant fiber bundle $\bar{f}_{j j^{\prime}}: \bar{f}_{j^{\prime}}\left(F\left(U_{j} \cap U_{j^{\prime}}\right)\right) \rightarrow \bar{f}_{j}\left(F\left(U_{j} \cap U_{j^{\prime}}\right)\right)$ such that $\bar{f}_{j j^{\prime}} \circ \bar{f}_{j^{\prime}}=\bar{f}_{j}$. Notice that this implies $\mathcal{O}_{j^{\prime}}(p) \subset \mathcal{O}_{j}(p)$ for any $p \in U_{j} \cap U_{j^{\prime}}$, and the $\mathfrak{N}$-orbit passing through $p \in M$ is then defined as $\mathcal{O}(p):=\cup_{j} \mathcal{O}_{j}(p)$. Clearly, we have a partition $M=\cup \mathcal{O}(p)$ into disjoint unions of $\mathfrak{N}$-orbits. We also define the rank of $\mathfrak{N}_{j}$ as rank $\mathfrak{N}_{j}:=\min _{p \in U_{j}} \operatorname{dim} \mathcal{O}_{j}(p)$, and the rank of $\mathfrak{N}$ as rank $\mathfrak{N}:=\min _{j} \operatorname{rank} \mathfrak{N}_{j}$. If $N_{j}=N$ for all $j$, we say that $\mathfrak{N}$ is a pure nilpotent structure, which is always the case when the collapsing sequence has uniformly bounded diameter, as shown in [36]. If each $N_{j}$ is abelian, we say that $\mathfrak{N}$ defines an $F$-structure.

Notice that on each $F\left(U_{j}\right)$, the fiber of $\bar{f}_{j}$ is diffeomorphic to the symmetric space $N_{j} / \Gamma_{j}$, on which the simply connected nilpotent Lie group $N_{j}$ acts (or equivalently, the sheaf of its Lie algebra maps homomorphically into the sheaf of vector fields tangent to the $\bar{f}_{j}$ fibers). We say that the $N$-structure $\mathfrak{N}$ is a nilpotent Killing structure compatible with a Riemannian metric $g^{\prime}$ on $M$, if the canonically induced metric $\bar{g}^{\prime}$ on $F(M)$ has its restriction $\left.\bar{g}^{\prime}\right|_{F\left(U_{j}\right)}$ for each $j$ being left invariant under the actions of $N_{j}$ (or equivalently, the sheaf of its Lie algebra maps homomorphically into the sheaf of $\bar{g}^{\prime}$-Killing vector fields tangent to the $\bar{f}_{j}$ fibers). Notice that the existence of the nilpotent Killing structure makes each fiber bundle map $\bar{f}_{j}: F\left(U_{j}\right) \rightarrow Y_{j}$ a Riemannian submersion. Moreover, the $\bar{g}^{\prime}$-Killing vector fields induced by each $N_{j}$ descends to $U_{j}$, defining $g^{\prime}$-Killing vector fields tangent to the $\mathfrak{N}_{j}$-orbits. When $\mathfrak{N}$ is pure, the partition of $M$ into the $\mathfrak{N}$-orbits then defines a (singular) Riemannian foliation, with leaves being the $\mathfrak{N}$-orbits; see [64] and Section 5.2 for more discussions.

The main achievement of [14] can now be stated as following; see also [75, Theorem 5.1].

Theorem 2.3 (nilpotent Killing structure, 1992). There is a $\varepsilon(m)>0$ such that for any positive $\varepsilon<\varepsilon(m)$, if $(M, g)$ is a complete Riemannian manifold with $\sup _{M}\left|\mathbf{K}_{g}\right| \leq 1$, and for any $x \in M$, $\left|B_{g}(x, 1)\right|<\varepsilon$, then the following hold:

1) there exists a regular $(\rho, k)$-round metric $g_{\varepsilon}$ such that $\left\|g-g_{\varepsilon}\right\|_{C^{1}(M, g)}<\Psi_{\mathrm{CFG}}(\varepsilon \mid m)$ holds for some $\Psi_{\mathrm{CFG}}(\varepsilon \mid m)>0$ with $\lim _{\varepsilon \rightarrow 0} \Psi_{\mathrm{CFG}}(\varepsilon \mid m)=0$;

2) there is a nilpotent Killing structure $\mathfrak{N}$ of positive rank, compatible with $g_{\varepsilon}$.

Here we say that a Riemannian metric $g$ is $(\rho, k)$-round, with $\rho$ and $k$ determined by $M$ and $\varepsilon$, if for any $p \in M$ there is an open neighborhood $U \subset M$ of $B_{g}(p, \rho)$, such that $U$ has a normal Riemannian covering $\tilde{U}$ with deck transformation group $\Lambda$, whose injectivity radius is uniformly bounded below by $\rho$, and there is an isometric action on $\tilde{U}$ by a Lie group $N$ 
with nilpotent identity component $N^{0}$, extending the deck transformations by $\Lambda$ with index bound $\left[N: N^{0}\right]=\left[\Lambda: \Lambda \cap N^{0}\right] \leq k$. In the presence of a compatible $N$-structure $\mathfrak{N}=\left\{\mathfrak{N}_{j}\right\}$, for any $p \in M$ there is some $U_{j}$ such that $B_{g}(p, \rho) \Subset U_{j}$, and $N^{0} \cong N_{j} / \mathbb{R}^{d}$ as Lie groups with $d=\operatorname{dim} G_{f_{j}(p)}$. Here $f_{j}: U_{j} \rightarrow X_{j}$ is the local singular fibration over the collapsing limit space $X_{j}$, and $G_{f_{j}(p)}$ is the isotropy group of $f_{j}(p) \in X_{j}$ as discussed previously. Since the identity component of $G_{f_{j}(p)}$ is a $d$-torus, its Lie algebra is indeed $\mathbb{R}^{d}$. Moreover, we have $\Lambda \cap N_{0} \cong q\left(\Gamma_{j}\right)$ with $q: N_{j} \rightarrow N^{0}$ being the natural quotient map.

Besides serving as a fundamental theorem of collapsing geometry with bounded sectional curvature, Theorems 2.2 and 2.3 have stimulated many exciting discoveries in Riemannian geometry. To name a few, low dimensional collapsed manifolds were investigated, for which some rationality conjectures of Cheeger and Gromov on the geometric invariants associated to collapsing were verified [73, 74], and the 4-dimensional case of Gromov's gap conjecture on the minimal volume was confirmed [72]; in [19, 20], the singular structures described in Theorem 2.2 were investigated, and the existence of a mixed polarized sub-structure (or a pure polarization when there is bounded covering geometry) was proven; in [8, 9], an Abelian structure has been constructed on very collapsed manifolds with bounded non-positive sectional curvature, verifying the Buyulo conjecture [5, 6] on $C r$-structure in general dimensions; in [31, 32, 70, 71], diffeomorphism stability and finiteness results have been established for very collapsed 2-connected manifolds; and in [7], one-parameter families of collapsing metrics with bounded sectional curvature have been constructed under the presence of the nilpotent Killing structures of positive rank, extending the previous works of Cheeger and Gromov [16].

\subsection{Almost flatness by weaker curvature assumptions}

Besides the far-reaching generalization of Gromov's almost flat manifold theorem to parametrized versions in the collapsing geometry with bounded sectional curvature, another direction of generalization is to weaken the curvature assumption (1.1) in Theorem 1.1.

In [30], Dai, Wei and Ye obtained a generalization for almost Ricci-flat manifolds whose conjugate radii are uniformly bounded below.

Theorem 2.4 (almost Ricci-flat manifolds, 1996). There is a uniform constant $\varepsilon(m) \in(0,1)$ such that if a closed $m$-dimensional Riemannian manifold $(M, g)$ with conjugate radii bounded below by 1 satisfies

$$
\operatorname{diam}(M, g)^{2} \max _{M}\left|\mathbf{R} \mathbf{c}_{g}\right|_{g} \leq \varepsilon^{2},
$$

then $M$ is diffeomorphic to an infranil manifold.

While this theorem seems to be expected directly from Gromov's almost flat manifold theorem, the proof actually requires the Ricci flow smoothing technique - to the authors' knowledge, this is the first instance where such a technique is employed for manifolds satisfying certain Ricci curvature bounds. This theorem is further generalized by Petersen, Wei and Ye in [69], where the regularity of the metrics is characterized by the harmonic $C^{0, \alpha}$ norm of the manifolds (for any $\alpha \in(0,1))$; see [69, Theorem 1.4], which we will not restate in this note for the sake of brevity.

A more striking conjecture of Gromov [40] predicted that even if only assuming almost nonnegative Ricci curvature, when the first Betti number of the manifold is equal to its dimension, then it has to be a flat $m$-torus. This conjecture is an effective version of the Bochner technique $[3,85]$ which asserts that a Ricci non-negatively curved manifold with maximal first Betti number (never exceeding the dimension) must be a flat torus, and is confirmed by Colding based on his volume continuity theorem [27]: 
Theorem 2.5 (Colding-Gromov gap theorem, 1997). There is a uniform constant $\varepsilon(m) \in(0,1)$ such that if a closed m-dimensional Riemannian manifold $(M, g)$ satisfies

$$
\operatorname{diam}(M, g)^{2} \mathbf{R c}_{g} \geq-\varepsilon^{2} g \quad \text { and } \quad b_{1}(M)=m,
$$

then $M$ is diffeomorphic to a flat torus.

This theorem can also be viewed as a quantitative version of the Cheeger-Gromoll splitting theorem [15] when we consider the universal covering space of the given manifold.

Remark 2.6. In [27], Colding proved that $M$ is homotopic to the torus when $\operatorname{dim} M=3$, and is homeomorphic to the torus when $\operatorname{dim} M>3$. The diffeomorphism statement was later proven in the joint work of Cheeger and Colding using the Reifenberg method; see [13, Appendix A].

Obviously, the weaker assumption of almost non-negative Ricci curvature by itself is not enough to detect the infranil manifold structure, and certain extra assumptions should be expected. The second-named author proposed in 2014 to study Riemannian manifolds with locally bounded Ricci covering geometry, considering those manifolds with Ricci curvature bounded below and non-collapsing local universal covering spaces. Much progress has been made since then (see Section 3), including the following almost flat manifold theorem due to the second-named author and his collaborators [49].

Theorem 2.7 (bounded Ricci covering geometry, 2018). Given $m, v>0$, there is a uniform constant $\varepsilon(m, v) \in(0,1)$ such that if a closed m-dimensional Riemannian manifold $(M, g)$ satisfies

$$
\operatorname{diam}(M, g)^{2} \mathbf{R c}_{g} \geq-\varepsilon^{2} g \quad \text { and } \quad\left|B_{\tilde{g}}(\tilde{p}, 1)\right| \geq v
$$

then $M$ is diffeomorphic to an infranil manifold. Here $\tilde{p}$ is any point in $\tilde{M}$, the universal covering space of $M$ equipped with the covering metric $\tilde{g}$.

Other types of weaker curvature assumptions include certain mixed curvature conditions considered by Kapovitch: in [55], the sectional curvature lower bound in Gromov's almost flat manifold theorem is weakened to a Bakry-Émery Ricci tensor lower bound.

Theorem 2.8 (mixed curvature conditions, 2019). There exists a uniform constant $\varepsilon(N) \in(0,1)$ such that if an m-dimensional $(m \leq N)$ weighted closed Riemannian manifold $\left(M, g, \mathrm{e}^{-f} \mathcal{H}^{m}\right)$ satisfies

$$
\operatorname{diam}(M, g)^{2} \max _{\wedge^{2} T M} \mathbf{K}_{g} \leq \varepsilon(N)^{2} \quad \text { and } \quad \operatorname{diam}(M, g)^{2}\left(\mathbf{R c}_{g}+\operatorname{Hess}_{f}\right) \geq-\varepsilon(N)^{2} g,
$$

then $M$ is diffeomorphic to an infranil manifold.

We notice here that the lower bound of the Bakry-Émery Ricci tensor $\mathbf{R c}_{g}+$ Hess $_{f}$ is essentially weaker than the corresponding Ricci curvature lower bound, which would imply almost non-negative sectional curvature in the context: as shown in [55, Lemma 8.1], on the 2-torus there is a sequence of Riemannian metrics satisfying the assumptions of the theorem, but the minimum of their sectional curvature (in $\wedge^{2} T \mathbb{T}^{2}$ ) have no finite lower bound. We also point out that it is more natural to consider the Bakry-Émery Ricci curvature lower bound in the collapsing setting: see the work of Lott [61]. 


\subsection{Collapsing manifolds with Ricci curvature bounded below}

More generally, one may consider the Gromov-Hausdorff limits of manifolds in the collection $\mathcal{M}_{\mathrm{Rc}}(m)$ of complete Riemannian $m$-manfiolds with the lowest eigenvalue of the Ricci tensor uniformly bounded below by $-(m-1)$. While the Gromov-Hausdorff limits of a sequence in $\mathcal{M}_{\mathrm{Rc}}(m)$ with an extra uniform volume lower bound (a non-collapsing sequence) has been thoroughly investigated through the works $[13,18]$, our understanding of a possibly collapsing sequence in $\mathcal{M}_{\mathrm{Rc}}(m)$ (i.e., a sequence without volume lower bound) is very limited. The ideal here is to develop a parallel theory as the collapsing geometry with bounded sectional curvature, and describe the geometry around points where the sectional curvature becomes unbounded.

Recall that in the classical theory, the collapsing with bounded sectional curvature is caused by the extra symmetry of the infranil fibers. It is therefore natural to focus on the local isometries of manifolds in $\mathcal{M}_{\mathrm{Rc}}(m)$. The understanding of such local isometry is encoded in the fibered fundamental group $\Gamma_{\delta}(p)$, defined for any $p \in M$ and $\delta \in(0,1)$ as

$$
\Gamma_{\delta}(p):=\text { Image }\left[\pi_{1}\left(B_{g}(p, \delta), p\right) \rightarrow \pi_{1}\left(B_{g}(p, 2), p\right)\right] .
$$

This group collects all loops contained in $B_{g}(p, \delta)$ and based at $p$, but that are allowed to deform within $B_{g}(p, 2)$. Concerning the structure of such groups, a key conjecture due to Gromov states that the fibered fundamental group is almost nilpotent. This conjecture is confirmed by Kapovitch and Wilking in [57].

Theorem 2.9 (generalized Margulis lemma, 2011). There are uniform constants $C(m) \geq 1$ and $\varepsilon(m) \in(0,1)$ such that for any $(M, g) \in \mathcal{M}_{\mathrm{Rc}}(m)$ and any $p \in M$, the fibered fundamental group $\Gamma_{\varepsilon(m)}(p)$ contains a nilpotent sub-group of nilpotency rank $\leq m$ and index $\leq C(m)$.

Based on a rescaling and contradiction argument, this theorem is strengthened by Naber and Zhang [66] when a geodesic ball is Gromov-Hausdorff close to a (lower) $k$-dimensional Euclidean $r$-ball (denoted by $\mathbb{B}^{k}(r)$ for any $r>0$ ): if $d_{\mathrm{GH}}\left(B_{g}(p, 2), \mathbb{B}^{k}(2)\right)<\varepsilon(m)$ for some $k \leq m$, then $\operatorname{rank} \Gamma_{\varepsilon(m)}(p) \leq m-k$. More significantly, they discovered certain topological conditions that guarantee a very strong regularity of the metric.

Theorem 2.10 ( $\varepsilon$-regularity for Ricci curvature, 2018). For any $\varepsilon>0$ there is a uniform constant $\delta(m, \varepsilon) \in(0,1)$ such that if $(M, g, p)$ is a pointed Riemannian m-manifold satisfying $\mathbf{R c}_{g} \geq-(m-1) g$ and $B_{g}(p, 2) \Subset B_{g}(p, 4)$, then for any normal covering $\pi:(W, \hat{p}) \rightarrow\left(B_{g}(p, 2), p\right)$ with $\pi(\hat{p})=p$, covering metric $\hat{g}$ and deck transformation group $G$, if

1) $d_{\mathrm{GH}}\left(B_{g}(p, 2), \mathbb{B}^{k}(2)\right)<\delta$, and

2) the almost nilpotent group $\widehat{G}_{\delta}(p):=\left\langle\gamma \in G: d_{\hat{g}}(\gamma \cdot \hat{p}, \hat{p})<2 \delta\right\rangle$ satisfies

$$
\operatorname{rank} \widehat{G}_{\delta}(p)=m-k,
$$

then for some $r \in(\delta, 1)$ it holds that

$$
d_{\mathrm{GH}}\left(B_{\hat{g}}(\hat{p}, r), \mathbb{B}^{m}(r)\right)<\varepsilon r .
$$

If we impose the extra assumption of a uniform Ricci curvature upper bound, this theorem directly proves a local fiber bundle theorem for domains collapsing to lower dimensional Euclidean balls; see [66, Proposition 6.6]. The same conclusion actually holds without the Ricci curvature upper bound, and this has been proven very recently by the authors based on the Ricci flow local smoothing techniques [54, Theorem 1.4]; see Theorem 4.9 in the next section. Notice that the assumption (1) in Theorem 2.10 is crucial in the original blow up argument, and the case of orbifold collapsing limit in [54, Theorem 1.4] is considerably more difficult; see Remark 4.10. Based on these results, the following theorem is recently proven in [53]. 
Theorem 2.11 (rigidity of the first Betti number, 2020). Given $D \geq 1$ and $v>0$, there is a uniform constant $\varepsilon(m, D, v) \in(0,1)$ such that if $(M, g) \in \mathcal{M}_{\mathrm{Rc}}(m)$ and $(N, h) \in \mathcal{M}_{\mathrm{Rm}}(k, D, v)$ satisfy $d_{\mathrm{GH}}(M, N)<\varepsilon(m, D, v)$ with $k \leq m$, then $b_{1}(M)-b_{1}(N) \leq m-k$. Moreover, if the equality holds, then $M$ is diffeomorphic to an $(m-k)$-torus bundle over $N$.

Here we notice that the assumptions of Theorem 2.10 are purely local, and a key difficulty in the proof was to localize the topological information encoded in the first Betti number, which is global in nature. Here we introduced the so-called pseudo-local fundamental group $\tilde{\Gamma}_{\delta}(p)$, which is defined for any $p \in M$ and $\delta \in(0,1)$ as $\tilde{\Gamma}_{\delta}(p):=\operatorname{Image}\left[\pi_{1}\left(B_{g}(p, \delta), p\right) \rightarrow \pi_{1}(M, p)\right]$. This concept provides a bridge linking $\pi_{1}(M, p)$ with $\Gamma_{\delta}(p)$. We also considered $H_{1}^{\delta}(M ; \mathbb{Z})$, generated by singular homology classes with a representation by a geodesic loop of length not exceeding $10 \delta$. Under the assumption $d_{\mathrm{GH}}(M, N)<\delta$, it is then shown that $b_{1}(M)-b_{1}(N)=\operatorname{rank} H_{1}^{\delta}(M ; \mathbb{Z})$; and for $\delta<\varepsilon(m)$ sufficiently small, generalizing the work of Colding and Naber [28] it is shown that $\operatorname{rank} H_{1}^{\delta}(M ; \mathbb{Z}) \leq \operatorname{rank} \tilde{\Gamma}_{\varepsilon(m)}(p) \leq m-k$ for any $p \in M$. Therefore, by Theorem 2.10 the assumption $b_{1}(M)-b_{1}(N)=m-k$ ensures the universal covering of $M$ to locally resemble the $m$-Euclidean space. We could then run the Ricci flow to obtain a regular metric that still collapses, and applying Theorem 2.1 we established the theorem - discussions on such Ricci flow smoothing technique will be in Section 4.

\section{$3 \quad$ Locally bounded Ricci covering geometry}

In this section, we discuss the program initiated by the second-named author around 2014 to investigate Riemannian manifolds with Ricci curvature bounded below and non-collapsing local universal covering spaces: its current status and its goal; see also the previous survey [76] by the second-named author for related discussions.

More precisely, we let $\widetilde{\mathcal{M}}_{\mathrm{Rc}}(m, \rho, v)$ denote the collection of complete $m$-dimensional Riemannian manifolds $(M, g)$ satisfying $\mathbf{R c}_{g} \geq-(m-1) g$ and for any $x \in M,\left|B_{\tilde{g}}(\tilde{x}, \rho)\right| \geq v$, where $\tilde{g}$ is the covering metric of the (incomplete) Riemannian universal covering space of $B_{g}(x, \rho)$, and $\tilde{x}$ is any point covering $x$. We call the quantity $\left|B_{\tilde{g}}(\tilde{x}, \rho)\right|$ the local rewinding volume of $x$ at scale $\rho$, denoted by $\widetilde{\operatorname{Vol}}_{g}(x, \rho)$. So roughly speaking, $\widetilde{\mathcal{M}}_{\mathrm{Rc}}(m, \rho, v)$ consists of manifolds with Ricci curvature and local rewinding volume (at scale $\rho$ ) uniformly bounded below, and we say that such manifolds have locally $(\rho, v)$-bounded Ricci covering geometry, or just locally bounded Ricci covering geometry.

The goal of studying locally bounded Ricci covering geometry is mainly to establish an analogue, for manifolds in $\widetilde{\mathcal{M}}_{\mathrm{Rc}}(m, \rho, v)$ and their (pointed) Gromov-Hausdorff limits, of the nilpotent structure theorey of Cheeger, Fukaya and Gromov (see Section 2.1).

As pointed out in the introduction, understanding the collapsing behaviors of manifolds of this type is a very natural and immediate step in our study of general collapsing phenomena of manifolds with Ricci curvature bounded below. If a Riemannian $m$-manifold has sectional curvature uniformly bounded by 1 in absolute value, then it has locally $(\rho(m), v(m))$-bounded Ricci covering geometry, where the constants only depend on the dimension $m$; see $[14,78]$.

\subsection{Singular infranil fiber bundles}

Recall that our ideal of study collapsing geometry with Ricci curvature bounded below is to recover, at least over most parts of the collapsing limit, the infranil fiber bundle structure. In the setting of collapsing with locally bounded Ricci covering geometry, this is indeed the case over the regular part of the collapsing limit. 
Theorem 3.1 (infranil fiber bundle, 2020). Given $m, k \in \mathbb{N}$ with $k<m, D \geq 1$ and $\rho, v_{1}, v_{2}>0$ there is a uniform constant $\delta\left(m, D, \rho, v_{1}, v_{2}\right)>0$ such that if $(M, g) \in \widetilde{\mathcal{M}}_{\mathrm{Rc}}\left(m, \rho, v_{1}\right)$ is $\delta$ Gromov-Hausdorff close to a manifold $(N, h) \in \mathcal{M}_{\mathrm{Rm}}\left(k, D, v_{2}\right)$, then there is a fiber bundle $f: M \rightarrow N$ which is also a $\Psi\left(\delta \mid m, D, \rho, v_{1}, v_{2}\right)$-Gromov-Hausdorff approximation, with whose fibers diffeomorphic to an $(m-k)$-dimensional infranil manifold, and whose structure group reduced to a generalized torus group as described in Theorem 2.2.

This theorem summarizes the contributions from [48] and [78]. In [48], the existence of the topological fiber bundle map $f$ is obtained using the canonical Reifenberg method in [18]. However, the infranil manifold structure of the fiber obtained in [78] is not a direct application of Theorem 2.7, since an $f$-fiber may have no uniform Ricci curvature lower bound. Considerations on the ambient geometry is instead carried out in [78] - this work, when restricting to the case of almost flat manifolds, provides for the first time an approach entirely different from the original one in [39, 79]; see also [77]. Notice that the arguments in $[48,78]$ are local and our statements here are valid even if $N$ is an open set in an $k$-dimensional manifold.

Besides substaintially generalizing the condition of collapsing with bounded sectional curvature, the condition of locally bounded Ricci covering geometry is also more general than the assumption of maximal nilpotency rank discussed in Theorem 2.10, which plays a key role in the proof of Theorem 2.11. In fact, even when collapsing with bounded sectional curvature occurs, the fibered fundamental group (or the pseudo-local fundamental group) based at the fiber over a corner point cannot have maximal nilpotency rank. Here we recall that for a collapsing limit space $X$, we have a singular fiber bundle $f: M \rightarrow X$ as described in Theorem 2.2, and we say $x \in X$ is a corner point if $\operatorname{dim} G_{x}>0$. We make, however, the following remark.

Remark 3.2. When the collapsing limit $X$ is a manifold, these three concepts of collapsing coincide: both (a) collapsing with locally bounded Ricci covering geometry, and (b) collapsing with Ricci curvature bounded below and maximal nilpotency rank at every point, imply the same collapsing infranil fiber bundle structure arising from collapsing with bounded sectional curvature, which clearly implies the cases (a) and (b).

For a general metric space arising as the Gromov-Hausdorff limits of manifolds in $\widetilde{\mathcal{M}}_{\mathrm{Rc}}(m, \rho, v)$, we propose the following conjecture; compare also Theorem 2.2 .

Conjecture 3.3 (singular nilpotent fibration). Suppose a sequence $\left\{\left(M_{i}, g_{i}\right)\right\} \subset \widetilde{\mathcal{M}}_{\mathrm{Rc}}(m, \rho, v)$ collapses to a lower dimensional compact metric space $X$, i.e., $M_{i}^{m} \stackrel{\mathrm{GH}}{\longrightarrow} X^{k}$ where the dimension is the sense of Colding-Naber [28]. Then for each $i$ sufficiently large, there is a singular fibration $f_{i}: M_{i} \rightarrow X$, such that a regular fiber is an infranil manifold, and a singular fiber is a finite quotient of an infranil manifold.

Some progress on understanding the structure of a Gromov-Hausdorff limit of a sequence in $\widetilde{\mathcal{M}}_{\mathrm{Rc}}(m, \rho, v)$ has already been made: see, e.g., [50]. Notice that if $\left\{\left(M_{i}, g_{i}\right)\right\} \subset \mathcal{M}_{\mathrm{Rc}}(m)$ and $M_{i} \stackrel{\mathrm{GH}}{\longrightarrow} X$ for some metric space $(X, d)$ with $\operatorname{diam}(X, d) \leq D$, then there is a renormalized measure $\nu$ on $X$, and by the work of Colding and Naber [28], there is a unique $k \leq m$ such that at $\nu$-a.e. point of $X$ any tangent cone is isometric to $\mathbb{R}^{k}$ : this $k$ will be denoted as $\operatorname{dim}_{\mathrm{CN}} X$, the dimension of $X$ in the sense of Colding-Naber. On the other hand, as a metric space one can talk about the Hausdorff dimension $\operatorname{dim}_{\mathrm{H}} X$. When $k=m$ we know that $\operatorname{dim}_{\mathrm{CN}} X=\operatorname{dim}_{\mathrm{H}} X$, but when $k<m$ this it remains an open question whether $\operatorname{dim}_{\mathrm{CN}} X=\operatorname{dim}_{\mathrm{H}} X$ : see, e.g., [56, Open Question 1.11]. However, for manifolds with locally bounded Ricci covering geometry, we have the following result [76].

Proposition 3.4. If $\left\{\left(M_{i}, g_{i}, p_{i}\right)\right\} \subset \widetilde{\mathcal{M}}_{\mathrm{Rc}}(m, \rho, v)$ and $M_{i} \stackrel{p \mathrm{GH}}{\longrightarrow} X$ with $(X, d, p)$ a pointed metric space, then there is some $k \leq m$ such that any tangent cone at any point of $X$ is a $k$ dimensional metric cone. Moreover, $\operatorname{dim}_{\mathrm{H}} X=k=\operatorname{dim}_{\mathrm{CN}} X$. 


\subsection{Almost maximal local rewinding volume}

In this sub-section, we consider an "extremal" case of locally bounded Ricci covering geometry: those manifolds in $\widetilde{\mathcal{M}}_{\mathrm{Rc}}(m, \rho, v)$ with almost maximal local rewinding everywhere; compare also [26]. Given $(M, g) \in \widetilde{M}_{\mathrm{Rc}}(m, \rho, v)$, since for any $x \in M$, the local covering metric has the same Ricci curvature lower bound as the original metric, the Bishop-Gromov volume comparison is in effect, implying that $\widetilde{\operatorname{Vol}}_{g}(\tilde{x}, \rho) \leq \Lambda_{\lambda(x)}^{m}(\rho)$, which denotes the volume of a geodesic $\rho$-ball in the space form of sectional curvature equal to $\lambda(x)$, with $\lambda(x)$ denoting the lowest eigenvalue of $\mathbf{R c}_{g}$ on $B_{g}(x, 2 \rho)$. When $M$ is compact, we let $\lambda(M, g):=\min _{x \in M} \lambda(x)$. If, however, we know that the local rewinding volume is almost maximal, then strong structural results have been obtained by the second-named author with his collaborators in [21, 22].

Theorem 3.5 (quantitative space form rigidity, 2019). Given $m, \rho, v>0$ with $\rho<1$ and a closed manifold $(M, g) \in \mathcal{M}_{\mathrm{Rc}}(m)$ whose Riemannian universal covering $(\tilde{M}, \tilde{g})$ has some $\tilde{p}_{0} \in \tilde{M}$ satisfying $\left|B_{\tilde{g}}\left(\tilde{p}_{0}, 1\right)\right|_{\tilde{g}} \geq v$, then we have the following:

1) if $\lambda(M, g)=1$ and $\inf _{x \in M} \widetilde{\operatorname{Vol}}_{g}(x, \rho) \geq(1-\varepsilon) \Lambda_{1}^{m}(\rho)$ for some uniform $\varepsilon \in(0,1)$ determined by $m, \rho$ and $v$, then $M$ is diffeomorphic to a spherical space form by a $\Psi(\varepsilon \mid m, \rho, v)$-isometry;

2) if $\lambda(M, g)=0, \operatorname{diam}(M, g) \leq 1$ and $\inf _{x \in M} \widetilde{\operatorname{Vol}_{g}}(x, \rho) \geq(1-\varepsilon) \Lambda_{0}^{m}(\rho)$ for some uniform $\varepsilon \in(0,1)$ determined by $m, \rho$ and $v$, then $M$ is isometric to a flat manifold; and

3) if $\lambda(M, g)=-1, \operatorname{diam}(M, g) \leq d$ and $\inf _{x \in M} \widetilde{\operatorname{Vol}_{g}}(x, \rho) \geq(1-\varepsilon) \Lambda_{-1}^{m}(\rho)$ for some uniform $\varepsilon \in(0,1)$ determined by $d, m, \rho$ and $v$, then $M$ is diffeomorphic to a hyperbolic manifold by a $\Psi(\varepsilon \mid m, \rho, v, d)$-isometry.

Note that manifolds satisfying items (1) or (2) in this theorem may be arbitrarily collapsed. In [22, Theorem D], a quantitative rigidity theorem for hyperbolic spaces (compare item (3) in Theorem 3.5) has been obtained for manifolds in $\mathcal{M}_{\mathrm{Rc}}(m)$ in terms of the volume entropy [59]. In fact, by the assumed uniform lower bound of the local rewinding volume, it is natural to ask if we can drop the non-collapsing assumption of the universal covering spaces:

Conjecture 3.6. Theorem 3.5 still holds for $(M, g) \in \mathcal{M}_{\mathrm{Rc}}(m)$ even if we do not assume the existence of $\tilde{p}_{0} \in \tilde{M}$ so that $\left|B_{\tilde{g}}\left(\tilde{p}_{0}, 1\right)\right|_{\tilde{g}} \geq v>0$.

In the case when a uniform Ricci curvature upper bound is additionally assumed, this conjecture has been verified by the second-named author and his collaborators in [21].

\section{Smoothing the locally collapsing metrics with Ricci curvature bounded below}

As mentioned in the introduction, the proofs of Theorems 2.1, 2.7, 2.8 and 2.11 all rely on the smoothing effect by globally running the Ricci flow. The Ricci flow with initial data $(M, g)$, first introduced by Hamilton [44] on closed 3-manifolds to deform a given Riemannian metric $g$ with positive Ricci curvature to a positive Einstein metric, is a smooth family of Riemannian metrics $g(t)$ on $M$ solving the following initial value problem for $t \geq 0$ :

$$
\left\{\begin{array}{l}
\partial_{t} g(t)=-2 \mathbf{R c}_{g(t)}, \\
g(0)=g
\end{array}\right.
$$


In harmonic coordinates, the Ricci flow becomes a non-linear heat-type equation for the metric tensor, and by the nature of the heat flows, notably Shi's estimates [80], a key effect of running Ricci flow is that the evolved metric has much improved regularity:

$$
\forall l \in \mathbb{N}, \exists C_{l}>0, \sup _{M}\left|\nabla^{l} \mathbf{R m}_{g(t)}\right|_{g(t)} \leq C_{l} t^{-1-l} .
$$

Here the constants $C_{l}$ depend on the dimension of $M$, as well as $\left\|\mathbf{R} \mathbf{m}_{g}\right\|_{C^{0}(M, g)}$. In fact, the finiteness of $\left\|\mathbf{R m}_{g}\right\|_{C^{0}(M, g)}$ guarantees the Ricci flow solution to (4.1) to exist for a definite amount of time determined by its value, even if $(M, g)$ is complete but non-compact.

In view of Shi's estimates, the Ricci flow also becomes a useful tool to smooth a given Riemannian metric by replacing the initially given metric $g$ with the evolved metric $g(t)$, whose regularity is controlled by (4.2) - in order to take advantage of such an estimate, a uniform lower bound of the Ricci flow existence time then becomes crucial. This method has been investigated in [30] for closed mainfolds, producing fruitful applications, such as the proofs of Theorem 2.4 and [66, Proposition 6.6] (see also [69]); but as the collapsing phenomenon may be observed locally on a geodesic ball, the localization of the Ricci flow existence results is usually necessary for the smoothing purpose. In this section, we will discuss the recent developments on the Ricci flow local smoothing techniques for collapsing initial data with Ricci curvature bounnded below.

\subsection{Local existence of the Ricci flow}

As shown in [54, Lemma 2.2], we could in fact start the Ricci flow locally on any Riemannian manifold with Ricci curvature bounded below.

Lemma 4.1. Given a complete Riemannian manifold $\left(M^{m}, g\right)$ with $\mathbf{R c}_{g} \geq-(m-1) g$ and let $K$ be a compact subset. For any $R>0$ there is a smooth family of Riemannian metrics $g(t)$ on $B_{g}\left(K, \frac{R}{4}\right)$ satisfying

$$
\left\{\begin{array}{l}
\partial_{t} g(t)=-2 \mathbf{R c}_{g(t)} \text { on } B_{g}\left(K, \frac{R}{4}\right) \times(0, T], \\
g(0)=g \text { on } B_{g}\left(K, \frac{R}{4}\right),
\end{array}\right.
$$

for some $T>0$, such that

$$
\forall t \in(0, T], \quad \sup _{B_{g}\left(K, \frac{R}{4}\right)}\left|\mathbf{R m}_{g(t)}\right|_{g(t)} \leq C t^{-1},
$$

where the positive constants $C$ and $T$ depend on $g, K$ and $R$.

This lemma is proven using Hochard's conformal transformation technique in [47, Section 6]. One can conformally blow the points near $\partial B_{g}(K, R)$ to infinity, obtaining a complete Riemannian metric $h$ defined on $B_{g}(K, R)$. Since $\overline{B_{g}(K, R)}$ is compact, the sectional curvature of $g$ is bounded, and thus so is the sectional curvature of the complete metric $h$ by making a good choice of the conformal factor. One could then rely on Shi's short time existence theorem to start a Ricci flow solution $h(t)$ with initial data $h(0)=h$. But since the conformal factor can be designed to be 1 on $B_{g}\left(K, \frac{R}{4}\right)$, one can view the Ricci flow solution $\left.h(t)\right|_{B_{g}\left(K, \frac{R}{4}\right)}$ as the local Ricci flow starting from the initial data $\left(B_{g}\left(K, \frac{R}{4}\right), g\right)$. We point out that the conformal factor could also be designed so that the scalar curvature and local isoperimetric constant lower bounds for $h$ are comparable to the ones for $g$.

As mentioned in the introduction, in order to use Ricci flow as a smoothing tool one needs a definite lower bound of the existence time. If the initial data has only Ricci curvature lower bound, then the existence time lower bound relies on certain non-collapsing condition - even if the actual $m$-dimensional initial data may collapse to a lower dimensional space, the local covering spaces are usually assumed to resemble the local $m$-dimensional Euclidean space. In this setting, our most recent result [54, Theorem 1.2] gives: 
Theorem 4.2. Given any $\alpha \in\left(0,10^{-1}\right)$, any positive $m, l \in \mathbb{N}$ and any $R \in(0,100)$, there are uniform constants $\delta_{O}(m, l, R, \alpha), \varepsilon_{O}(m, \alpha) \in(0,1)$ to the following effect: let $K$ be a compact and connected subset of $\left(M^{m}, g\right)$, an m-dimensional Riemannian manifold with $\mathbf{R c}_{g} \geq-(m-1) g$, suppose for some $k \leq m$ and $\delta \leq \delta_{O}$ it satisfies for any $p \in B_{g}(K, R)$ the following assumptions:

1) there are a finite group $G_{p}<\mathrm{O}(k)$ with $\left|G_{p}\right| \leq l$ and a $\phi_{p} \in \operatorname{Hom}\left(\pi_{1}\left(B_{g}(K, R), p\right), G_{p}\right)$ which is surjective,

2) $d_{\mathrm{GH}}\left(B_{g}\left(p, 4^{-1} R\right), \mathbb{B}^{k}\left(4^{-1} R\right) / G_{p}\right)<\delta$, and

3) $\operatorname{rank} \tilde{\Gamma}_{\delta}(p)=m-k$,

then there is a Ricci flow solution with initial data $\left(B_{g}\left(K, \frac{R}{4}\right), g\right)$, existing for a period no shorter than $\varepsilon_{O}^{2}$, and with curvature control

$$
\forall t \in\left(0, \varepsilon_{O}^{2}\right], \quad \sup _{B_{g}\left(K, \frac{R}{4}\right)}\left|\mathbf{R m}_{g(t)}\right|_{g(t)} \leq \alpha t^{-1}+\varepsilon_{O}^{-2} .
$$

Here the notation $\tilde{\Gamma}_{\delta}(p):=\operatorname{Image}\left[\pi_{1}\left(B_{g}(p, \delta), p\right) \rightarrow \pi_{1}\left(B_{g}(K, R), p\right)\right]$ is the pseudo-local fundamental group for $B_{g}(K, R)$, containing all geodesic loops in $B_{g}(p, \delta)$ with base point $p$, and are allowed to be deformed within the entire $B_{g}(K, R)$.

Theorem 4.2 is proven roughly as following: by conditions (1) and (2), for each $p \in B_{g}\left(K, \frac{3}{4} R\right)$ we can find a finite normal covering of $B_{g}\left(p, \frac{R}{4}\right)$, so that it is $\Psi(\delta)$-Gromov-Hausdorff close to $\mathbb{B}^{k}\left(\frac{R}{4}\right)$; this condition, together with the nilpotency rank assumption in condition (3), enable us to show that the isoperimetric constant in a fix-sized geodesic ball around any point of the universal covering space of $B_{g}\left(K, \frac{3}{4} R\right)$ is very close to the $m$-Euclidean isoperimetric constant. By the design of the conformal factor, such almost locally Eucliean property is almost preserved under the conformal transformation, and together with the (relaxed) scalar curvature lower bound of the conformally transformed metric, it enables us to apply Perelman's pseudo-locality theorem (see the next sub-section) to bound the Ricci flow existence time from below.

Theorem 4.2 characterizes the "almost locally Euclidean covering space" assumption via algebraic conditions, i.e., the maximality of the rank of the pseudo-local fundamental groups and the existence of a surjective homomorphism of the local fundamental group onto the orbifold groups. One could also directly assume that the local universal covering space resembles the $m$-Euclidean space up to a fixed scale, defining the so-called $(\delta, \rho)$-Reifenberg points. For any $p \in M^{m}$, we say it is a $(\delta, \rho)$-Reifenberg point, if for any lift $\tilde{p}$ of $p$ in the Riemannian universal covering space of $B_{g}(p, \rho)$,

$$
\forall r \in(0, \rho], \quad r^{-1} d_{\mathrm{GH}}\left(B_{\tilde{g}}(\tilde{p}, r), \mathbb{B}^{m}(r)\right) \leq \delta .
$$

This definition essentially appears in the work [49] of the second-named author and his collaborators, and is for the purpose of defining the concept of Ricci bounded local covering geometry. Notice that with bounded Ricci curvature, if $p$ is a $(\delta, 2 \rho)$-Reifenberg point for $\delta$ sufficiently small, then $B_{\tilde{g}}(\tilde{p}, \rho)$ has a uniform lower bound on the $C^{1, \frac{1}{2}}$ harmonic radius. On the other hand, one could always run the Ricci flow locally around a $(\delta, \rho)$-Reifenberg point for a definite amount of time.

Theorem 4.3. For any $\alpha, \rho \in(0,1)$ there are uniform $\delta(m, \alpha, \rho) \in(0,1)$ and $\varepsilon(m, \alpha, \rho) \in(0,1)$ such that if $(M, g)$ is a complete Riemannian manifold with $\mathbf{R c}_{g} \geq-(m-1) g$, and $p \in M$ is $a(\delta, 2 \rho)$-Reifenberg point, then there is a Ricci flow solution with initial data $\left(B_{g}(p, \rho), g\right)$, that exists up to time $\varepsilon^{2}$ and for any $t \in\left[0, \varepsilon^{2}\right]$, the curvature satisfies

$$
\sup _{B_{g}(p, \rho)}\left|\mathbf{R m}_{g(t)}\right| \leq \alpha t^{-1}
$$


Sketch of proof. We could always start the Ricci flow $h(t)$ by Lemma 4.1 on $B_{g}(p, 2 \rho)$. Moreover, we could make sure that the initial data satisfies $\left.\left.h(0)\right|_{B_{g}(p, \rho)} \equiv g\right|_{B_{g}(p, \rho)}$. We only need to bound the existence time of the Ricci flow from below, which in turn relies on showing that the isoperimetric constant at any point of the covering space is almost Euclidean on a fixed scale. This is proven in [10], thanks to the definition of the $(\delta, \rho)$-Reifenberg property, as long as $\delta$ is sufficiently small. One then relies on Perelman's pseudo-locality theorem to prove that the flow exists for a definite period of time.

Let us also mention that in the case of Kapovitch's mixed curvature condition, it can be shown that the assumptions of Theorem 2.8 guarantees the local universal covering space at a point of the manifold to be almost locally Euclidean, via the aspherical theorem [55, Theorem 5.3].

\subsection{The pseudo-locality theorem}

In all the results discussed above, once the almost locally Euclidean condition for the local covering space is verified, the lower bound of the existence time of the Ricci flow is guaranteed by Perelman's pseudo-locality theorem, stated in its various forms as following:

Theorem 4.4 (Perelman's pseudo-locality theorem). For any $\alpha \in(0,1)$, there are uniform positive constants $\varepsilon_{P}=\varepsilon_{P}(m, \alpha)$ and $\delta_{P}=\delta_{P}(m, \alpha)$ such that if $(M, g)$ is a Ricci flow solution define for $t \in[0, T]$ with each time slice $(M, g(t))$ being a complete Riemannian manifold, and if one of the conditions holds for $p \in M$ :

1) $\mathbf{R}_{g(0)} \geq-1$ on $B_{g(0)}(p, 1)$ and $I_{B_{g(0)}(p, 1)} \geq\left(1-\delta_{P}\right) I_{m}$, or

2) $\mathbf{R c}_{g(0)} \geq-\delta_{P} g(0)$ on $B_{g(0)}(p, 1)$ and $\left|B_{g(0)}(p, 1)\right|_{g(0)} \geq\left(1-\delta_{P}\right) \omega_{m}$,

where $I_{m}$ and $\omega_{m}$ stands for the isoperimetric constant and volume of the $m$-Euclidean unit ball, respectively, and $I_{\Omega}$ denotes the isoperimetric constant for the domain $\Omega \subset M$, then

$$
\forall t \in\left(0, \varepsilon_{P}^{2}\right], \quad \sup _{B_{g(t)}\left(p, \varepsilon_{P}\right)}\left|\mathbf{R m}_{g(t)}\right|_{g(t)} \leq \alpha t^{-1}+\varepsilon_{P}^{-2} .
$$

The theorem originates from Perelman's work for closed manifolds satisfying condition (1) above; see [68, Theorem 10.1]. Later a version for complete non-compact manifolds was obtained by Chau, Tam and $\mathrm{Yu}$; see [11, Theorem 8.1]. The theorem with condition (2) was proven by Tian and the third-named author for closed manifolds in [82, Proposition 3.1], and its counterpart for complete non-compact data appears in the recent work of the authors' in [53, Proposition 6.1]. We point out that all the later works essentially follow Perelman's original idea and arguments.

In the proofs of Theorems 4.2, 4.3 and [55, Theorem 7.2], the almost locally Euclidean property for the local covering spaces checked before allows us to apply the pseudo-locality theorem to the covering flow and obtain a uniform lower bound on the existence time of the Ricci flow started via Lemma 4.1: if the existence time $T$ of the Ricci flow were shorter than $\varepsilon_{P}^{2}$, then for some sequence $t_{i} \nearrow T$ we could observe points $x_{i} \in M$ such that $\lim _{t_{i} \rightarrow T}\left|\mathbf{R m}_{h\left(t_{i}\right)}\right|_{h\left(t_{i}\right)}\left(x_{i}\right)=\infty$; especially, we will get $\left|\mathbf{R m}_{h\left(t_{i}\right)}\right|_{h\left(t_{i}\right)}\left(x_{i}\right)>2 \alpha T^{-1}+\varepsilon_{P}^{-2}$ for all $i$ large enough, contradicting the conclusion (4.3) since $T>0$ is fixed.

Heuristically speaking, Perelman's pseudo-locality theorem tells that the Ricci flow locally "preserves" the almost Euclidean parts of the manifolds. And it is natural to wonder if the initial data locally approaches lower dimensional Euclidean spaces, whether a pseudo-locality type theorem still holds. In fact, after proving a version of the pseudo-locality theorem [68, Theorem 10.3], Perelman asked:

"A natural question is whether the assumption on the volume of the ball is superfluous."

We notice however, that there are examples (see, e.g., $[63,49]$ ) that show the direct removal of the initial local volume non-collapsing assumption is fatal: 
Example 4.5 (Topping). Let $M_{\delta}$ denote the smooth manifold obtained from capping off the $\delta$-thin cylinder $\delta \mathbb{S}^{1} \times[-1,1]\left(\mathbb{S}^{1}\right.$ is identified with the unit circle in $\mathbb{C}$ with base point $\left.1 \in \mathbb{C}\right)$ by two discs of radius approximately $\frac{\pi}{2} \delta$ and slightly smoothing near the ends of the cylinder. The natural metric $g_{\delta}$ can be easily made to have non-negative sectional curvature. It is also obvious that around the base point $p_{\delta}=(\delta, 0)$ of $M_{\delta}$, the geodesic ball $B_{g_{\delta}}\left(p_{\delta}, \frac{1}{2}\right)$ is flat and is $\delta$-Gromov-Hausdorff close to $\left(-\frac{1}{2}, \frac{1}{2}\right)$. However, the Ricci flow starting from $M_{\delta}$ exits only for a period determined by the area of $M_{\delta}$, which is proportional to $\delta$. Therefore, as $\delta \searrow 0$, a curvature bound of the form (4.3) cannot be obtained for any uniform $\varepsilon>0$.

Fortunately, in many natural settings, the scalar curvature is indeed uniformly bounded along the Ricci flow, and here we raise the following

Conjecture 4.6. Given $\alpha \in(0,1)$, there are positive constants $\delta=\delta(m, \alpha)$ and $\varepsilon=\varepsilon(m, \alpha)$ such that if $(M, g)$ is an m-dimensional Ricci flow solution on $[0, T]$ with each of whose time slices being complete, and for some $p \in M$ it satisfies

$$
\sup _{B_{g(0)}(p, 1)}\left|\mathbf{R m}_{g(0)}\right|_{g(0)} \leq 1, \quad d_{\mathrm{GH}}\left(B_{g(0)}(p, 1), \mathbb{B}^{k}(1)\right) \leq \delta, \quad \sup _{M \times[0, T]}\left|\mathbf{R}_{g(t)}\right| \leq 1,
$$

then we have for any $t \in\left[0, \varepsilon^{2}\right]$, the curvature bound

$$
\sup _{B_{g(t)}(p, \varepsilon)}\left|\mathbf{R m}_{g(t)}\right|_{g(t)} \leq \alpha t^{-1}+\varepsilon^{-2} .
$$

\subsection{Distance distortion estimates}

Once the Ricci flow exists for a definite amount of time, for the purpose of smoothing, it is of key importance to compare the initial metric with the evolved metric. In general, the distance distortion estimate for Ricci flows is of central importance in the understanding of the geometry along the Ricci flows, and we refer the readers to [1, 2, 23, 24, 25, 45, 52, 82] for previous works on this topic in various settings. Very recently, based on the previous contributions, especially the local entropy theory developed in [84], the distance distortion estimate for collapsing initial data [52], and the Hölder distance estimate for non-collapsing initial data in [49], we obtain the following Hölder distance estimate for collapsing initial data [54, Theorem A.1]:

Theorem 4.7. Given a positive integer $m$, positive constants $\bar{C}_{0}, C_{R}, T \leq 1$ and $\alpha \in(0,1)$, there are constants $C_{D}\left(\bar{C}_{0}, C_{R}, m\right) \geq 1$ and $T_{D}\left(\bar{C}_{0}, C_{R}, m\right) \in(0, T]$ such that for an m-dimensional complete Ricci flow $(M, g(t))$ defined for $t \in[0, T]$, if for some $x_{0} \in M$ and any $t \in[0, T]$ we have

$$
\begin{aligned}
& \mathbf{R}_{g(0)} \geq-C_{R} \quad \text { in } B_{g(0)}\left(x_{0}, 10\right), \\
& \left|\mathbf{R c}_{g(t)}\right|_{g(t)} \leq \alpha t^{-1} \quad \text { in } B_{g(t)}\left(x_{0}, 10+\sqrt{t}\right),
\end{aligned}
$$

and the initial metric has a uniform bound $\bar{C}_{0}$ on the doubling and Poincaré constant for the geodesic ball $B_{g(0)}\left(x_{0}, 10\right)$, then for any $x, y \in B_{g(0)}\left(x_{0}, \sqrt{T_{D}}\right)$ and $t \in\left[0, T_{D}\right]$, we have

$$
C_{D}^{-1} d_{g(0)}(x, y)^{1+2 \alpha} d_{g(0)}(x, y) \leq d_{g(t)}(x, y) \leq C_{D} d_{g(0)}(x, y)^{1-2 \alpha} .
$$

Notice that the curvature assumption is natural (in view of the pseudo-locality theorem) and the comparison with the initial time slice is the key difficulty - for positive time slices the Ricci curvature bound makes the estimate trivial. Another handy distance distortion estimate for the application of smoothing the collapsing initial is the following 
Lemma 4.8. For any $\alpha \in(0,1)$, there is a positive quantity $\Psi_{D}(\alpha \mid m)$ with $\lim _{\alpha \rightarrow 0} \Psi_{D}(\alpha \mid m)=0$, such that under the assumption of Theorem 4.2 or Theorem 4.3, for any $x, y \in B_{g}(p, 2)$ and any $t \in\left(0, \varepsilon_{P}^{2}(m, \alpha)\right]$, if $d_{g}(x, y) \leq \sqrt{t}$, then we have

$$
\left|d_{g(t)}(x, y)-d_{g}(x, y)\right| \leq \Psi_{D}(\alpha \mid m) \sqrt{t} .
$$

This lemma is a slight re-wording of [49, Lemma 1.11], which concerns non-collapsing initial data. See also [54, Lemma 4.1] for a proof.

\subsection{Applications of the Ricci flow local smoothing technique}

With the Ricci flow smoothing tool kit at hand (the flow existence time lower bounds and the distance distortion estimates), we could in many cases reduce our consideration of collapsing manifolds with Ricci curvature bounded below to the classical collapsing geometry with bounded sectional curvature.

Locally, one could obtain infranil fiber bundle structure around points where the Ricci flow smoothing results (Theorems 4.2 and 4.3) apply:

Theorem 4.9. There is a positive constant $\delta=\delta(m)$ such that if $(M, g)$ is an $m$-dimensional complete Riemannian manifold with $\mathbf{R c}_{g} \geq-(m-1) g$, then for any $p \in M$ which has a geodesic ball satisfying

$$
d_{\mathrm{GH}}\left(B_{g}(p, 2), \mathbb{B}^{k}(2)\right)<\delta
$$

and one of the following conditions:

1) $p$ is a $(\delta, 2)$-Reifenberg point [49], or

2) $\operatorname{rank} \Gamma_{\delta}(p)=m-k[54]$,

there is an open neighborhood $U$ of $p$ such that $B_{g}(p, 1-2 \delta) \Subset U \Subset B_{g}(p, 1+2 \delta)$, and $U$ is diffeomorphic to an infranil fiber bundle over $\mathbb{B}^{k}(1)$, with the extrinsic diameter of the fibers bounded above by $2 \delta$.

Remark 4.10. In fact, as shown in [54, Theorem 1.4], there is a positive constant $\delta=\delta(m, l)<1$ such that if $p \in M$ has a geodesic ball satisfying $d_{\mathrm{GH}}\left(B_{g}(p, 2), \mathbb{B}^{k}(2) / G\right)<\delta$ for some $G<\mathrm{O}(k)$ with $|G| \leq l, \operatorname{rank} \Gamma_{\delta}(p)=m-k$, and there exists a surjective $\phi \in \operatorname{Hom}\left(\Gamma_{\delta}(p), G\right)$, then the same infranil fiber bundle structure over the orbifold neighborhood $\mathbb{B}^{k}(1) / G$ can be obtained. One can of course replace the assumption on the nilpotency rank with the Reifenberg property as in item (1). See also [36, Section 7] for related concepts.

Theorem 4.9 with condition (2) generalizes a local fiber bundle result due to Naber and Zhang [66, Proposition 6.6] from the case of manifolds with bounded Ricci curvature to manifolds with Ricci curvature only bounded from below. It is also a localization of [49, Theorem B]. To prove this theorem, we first notice that for any $\alpha \in(0,1)$ sufficiently small the assumptions enable us to run a Ricci flow with the local initial data for a definite period of time, and obtain a smoothing metric $g\left(\varepsilon^{2}\right)$ which is regular; by the distance distortion estimate Lemma 4.8, we know that up to scale $\varepsilon$, the original metric structure defined by $g$ is $\Psi(\alpha)$-Gromov-Hausdorff close to the metric structure defined by $g(\varepsilon)$; therefore, since the domain $\left(B_{g}(p, 2), g\right)$ is $\delta$-Gromov-Hausdorff close to $\mathbb{B}^{k}(1)$, we know that the domain $\left(B_{g}\left(p, \frac{3}{2}\right), g\left(\varepsilon^{2}\right)\right)$ is $\delta+\Psi(\alpha) \varepsilon$-Gromov-Hausdorff close to $\mathbb{B}^{k}(1)$ on scales up to $\varepsilon$; but then the regularity of the metric $g\left(\varepsilon^{2}\right)$ allows us to appeal to the classical theory of collapsing geometry [14, Theorem 2.6] with bounded sectional curvature to obtain the infranil fiber bundle structure over $\mathbb{B}^{k}(1)$. 
Here we would like to emphasize that the classical theorems (e.g., Theorems 2.1 and 2.3, as well as [14, Theorem 2.6]) on collapsing with bounded sectional curvature essentially describe a gap phenomenon, rooted back in Gromov's almost flat manifold theorem (Theorem 1.1): when the manifold is sufficiently Gromov-Hausdorff close to a lower dimensional space, then the manifold itself already acquires some non-trivial symmetry. Such a gap phenomenon allows us to slightly perturb the given metric locally to one with much better regularity, but remains to be sufficiently collapsed (in the metric sense) so that the symmetry structure could still be observed.

The Ricci flow local smoothing results can also help with proving global results when the collapsing limit is singular. In particular, we make the following

Conjecture 4.11. Given $D \geq 1, m, l \in \mathbb{N}$ and $\iota>0$ there is an $\varepsilon(m, l, \iota)>0$ such that if $(M, g) \in \mathcal{M}_{\mathrm{Rc}}(m, D)$ and an $(l, \iota)$-controlled $k$-dimensional Riemannian orbifold $\left(X, d_{X}\right)$ satisfy the conditions $d_{\mathrm{GH}}(M, X)<\varepsilon$ and $b_{1}(M)-b_{1}(X)=m-k$, then $M$ is a torus bundle over $X$.

Here by saying the Riemannian orbifold $\left(X, d_{X}\right)$ is $(l, \iota)$-controlled we mean that for any $x \in X, B_{d_{X}}(x, \iota) \equiv \mathbb{B}^{k} / G_{x}$ with the order of the orbifold group $G_{x}<\mathrm{O}(k)$ bounded above by $l$.

\section{Collapsing Ricci-flat Kähler metrics with bounded curvature}

Combining the classical theory of collapsing geometry with bounded curvature and the Ricci flow smoothing technique, we make some attempts to understand open Question 1.2.

If $(M, \bar{g}, \bar{J})$ is a closed Calabi-Yau manifold with $\bar{g}$ being a Ricci-flat Kähler metric, then by the work of Dai, Wang and Wei [29], there is a $C^{k}$-stability radius (in the space of Riemannian metrics on $M) \bar{\eta}_{1}>0$ determined by $\bar{g}$, such that the Ricci flow initiated from any Riemannian metric $g \in B_{C^{k}(M, \bar{g})}\left(\bar{g}, \bar{\eta}_{1}\right)$ converges to another Ricci-flat Kähler metric in $B_{C^{k}(M, \bar{g})}\left(\bar{g}, \bar{\eta}_{0}\right)$, with $\bar{\eta}_{1}<\bar{\eta}_{0}<1$. On the other hand, if $(M, \bar{g})$ is sufficiently volume collapsing with bounded sectional curvature, i.e., the volume $|M|_{\bar{g}}<\varepsilon(m)$ as in Theorem 2.3, then we are able to perturb $\bar{g}$ to some nearby metric which is invariant under the extra symmetry that causes collapsing. If now $|M|_{\bar{g}}<\varepsilon$ with $\varepsilon$ so small that the constant $\Psi_{\mathrm{CFG}}(\varepsilon \mid m)$ is small enough to guarantee the convergence of the Ricci flow starting from the approximating metric, then the flow will enable us to find a Ricci-flat Kähler metric compatible with the symmetry. This is the content of the following theorem.

Theorem 5.1. Given a closed Kähler manifold $(M, \bar{g}, \bar{J})$ equipped with a Calabi-Yau metric $\bar{g}$ such that $\max _{\wedge^{2} T M}\left|\mathbf{K}_{\bar{g}}\right| \leq 1$, there is a constant $\beta(\bar{g})>0$ such that if $\beta(\bar{g})<1$, then

1) there is a Ricci-flat Kähler metric $g$ (together with a compatible complex structure $J$ ), such that $\|g-\bar{g}\|_{C^{k}(M, \bar{g})}<\bar{\eta}_{0}$ for some $\bar{\eta}_{0} \in(0,1)$ solely determined by $\bar{g}$;

2) there are a Ricci-flat orbifold $X$ and a Riemannian submersion $f: M \rightarrow X$ with respect to $g$, such that the fibers are totally geodesic tori (see [36, Section 7] for related definitions), and $g$ is invariant under the trous action.

Here we have $\beta(\bar{g}):=|M|_{\bar{g}} \bar{\eta}_{3}^{-1}$, with $\bar{\eta}_{3}$ to be determined as following: notice that Cheeger, Fukaya and Gromov's approximating metric is only in a $C^{1}$ neighborhood of $\bar{g}$, but Dai, Wang and Wei's stability result requires much higher regularity for the neighborhood; therefore we develop a regularity improvement tool (Theorem 5.2), which finds a $C^{0}$ neighborhood of $\bar{g}$, denoted by $B_{C^{0}(M, \bar{g})}\left(\bar{g}, \bar{\eta}_{2}\right)$, where the Ricci flow exists forever and converges to a Ricci-flat Kähler metric; here $\bar{\eta}_{3}$ is defined so that if $|M|_{\bar{g}}<\bar{\eta}_{3}$, then $\Psi_{\mathrm{CFG}}\left(\bar{\eta}_{3} \mid m\right) \leq \frac{1}{2} \bar{\eta}_{2}$.

While the assumption on the volume collapsing is rather strong, in that $\beta(\bar{g})$ depends on the specific Kähler manifold, the existence of an invariant critical metric drastically reduces 
the topological complexity of the manifold: it is a torus bundle over a Ricci-flat orbifold. The invariant metric allows us to apply the O'Neill's formula [67], together with the central density (see [51, Section 5]) of the collapsing structure to rule out the so-called corner singularities of the collapsing limit space, and following the arguments in $[62,65]$ we can show that the collapsing fibers must be tori and the fibration must locally be a Riemannian product, implying the Ricci-flatness of the collapsing limit.

\subsection{Existence of invariant Ricci-flat Kähler metric}

In this sub-section we prove the first claim in Theorem 5.1. By the stability result [29] of Dai, Wang and Wei for Ricci-flat Kähler metrics, we know that for the Calabi-Yau manifold $(M, \bar{g}, \bar{J})$, there are some positive constants $\bar{\eta}_{1}<\bar{\eta}_{0}<1$, both determined by $\bar{g}$, such that if $g$ is another smooth Riemannian metric with $\|g-\bar{g}\|_{C^{k}(M, \bar{g})}<\bar{\eta}_{1}$, then the Ricci flow with initial data $g$ exists for all time and converges to a Ricci-flat Kähler metric in $B_{C^{k}(M, \bar{g})}\left(\bar{g}, \bar{\eta}_{0}\right)$. Here $k:=\left\lceil\bar{\eta}_{1}^{-1}\right\rceil$ is solely determined by $\bar{g}$. Since $k$ may be a very large number, our first priority is to prove the following regularity improvement result.

Theorem 5.2. There is a constant $\bar{\eta}_{2} \in\left(0, \bar{\eta}_{1}\right)$ determined by $\bar{g}$, such that if $\|g-\bar{g}\|_{C^{0}(M, \bar{g})}<\bar{\eta}_{2}$, then the Ricci flow starting from $g$ exists for all time and converges to a Ricci-flat Kähler metric in $B_{C^{k}(M, \bar{g})}\left(\bar{g}, \bar{\eta}_{0}\right)$.

Before proving Theorem 5.2, we state the following slight variant of Lemma 4.8:

Lemma 5.3. There is a positive constant $\bar{\eta}_{2}^{\prime}<1$ determined by $\bar{g}$ such that if $g \in B_{C^{0}(M, \bar{g})}\left(\bar{g}, \bar{\eta}_{2}^{\prime}\right)$, and the Ricci flow $g(t)$ with initial data $g$ satisfies $\max _{M}\left|\mathbf{R c}_{g(t)}\right|_{g(t)}<\alpha t^{-1}$ for $t \in[0, T)$ and some $\alpha \in(0,1)$, then there is a $\Psi_{D}^{\prime}(\alpha \mid \bar{g})>0$ with $\lim _{\alpha \rightarrow 0} \Psi_{D}^{\prime}(\alpha \mid \bar{g})=0$ such that for any $x, y \in M$ with $d_{g}(x, y) \leq \sqrt{t}$, we have

$$
\left|d_{g}(x, y)-d_{g(t)}(x, y)\right| \leq \Psi_{D}^{\prime}(\alpha \mid \bar{g}) \sqrt{t} .
$$

The proof of this lemma is the same as that of [22, Lemma 2.10]: in the contradiction argument involved, the rescaling limit will be exactly $\mathbb{R}^{n}$ as the quantity $\Psi_{D}^{\prime}(\alpha \mid \bar{g})$ depends on $\bar{g}$. With such distance distortion estimate at our disposal, we now prove the $C^{0}$ stability around a stable Ricci-flat Kähler metric.

Proof of Theorem 5.2. We notice that since $M$ is a closed manifold, for any smooth Riemannian metric $g$ there is a Ricci flow solution with initial data $g$. Moreover, for any $\alpha>0$, there is alway some $\varepsilon>0$ (depending on $\alpha$ and $(M, g)$ ) such that the following curvature bound is valid for $t \in\left(0, \varepsilon^{2}\right]$ :

$$
\left\|\mathbf{R m}_{g(t)}\right\|_{C^{0}(M, g(t))} \leq \alpha t^{-1} .
$$

We now make the following claim regarding the Ricci flow solution:

Claim 5.4. There are some $\alpha \in(0,1)$ and $\delta \in\left(0, \bar{\eta}_{2}^{\prime}\right)$ depending on $(M, \bar{g})$ such that if

$$
\|g-\bar{g}\|_{C^{0}(M, \bar{g})}<\delta \quad \text { and } \quad \forall t \leq \varepsilon^{2}, \quad\left\|\mathbf{R m}_{g(t)}\right\|_{C^{0}(M, g(t))} \leq \alpha t^{-1},
$$

then $\left\|g\left(\varepsilon^{2}\right)-\bar{g}\right\|_{C^{k}(M, \bar{g})}<\bar{\eta}_{1}$.

Proof of the claim. We now prove this claim via a contradiction argument: if the theorem fails, we may find a sequence of smooth Riemannian metrics $g_{i}$ on $M$ and sequences of positive 
numbers $\alpha_{i} \rightarrow 0$ and $\delta_{i} \rightarrow 0$, such that $\left\|g_{i}-\bar{g}\right\|_{C^{0}(M, \bar{g})}=\delta_{i} \rightarrow 0$ as $i \rightarrow \infty$, the Ricci flow solutions $g_{i}(t)$ satisfy

$$
\forall t \leq \varepsilon_{i}^{2}, \quad\left\|\mathbf{R m}_{g_{i}(t)}\right\|_{C^{0}\left(M, g_{i}(t)\right)} \leq \alpha_{i} t^{-1} .
$$

for some $\varepsilon_{i} \in(0,1)$, but $\left\|g_{i}\left(\varepsilon_{i}^{2}\right)-\bar{g}\right\|_{C^{k}(M, \bar{g})} \geq \bar{\eta}_{1}$ for all $i$ sufficiently large.

From the contradiction hypothesis we may find points $p_{i} \in M$ such that for all $i$ large enough,

$$
\left|g_{i}\left(\varepsilon_{i}^{2}\right)-\bar{g}\right|_{C^{k}}\left(p_{i}\right) \geq \bar{\eta}_{1} .
$$

On the other hand, by the curvature control (5.1) and Shi's estimates we have

$$
\forall l \in \mathbb{N}, \quad\left\|\nabla^{l} \mathbf{R m}_{g_{i}\left(\varepsilon_{i}^{2}\right)}\right\|_{C^{0}\left(M, g_{i}\left(\varepsilon_{i}^{2}\right)\right)} \leq C_{l} \varepsilon_{i}^{-2-2 l},
$$

with $C_{l}>0$ depending only on $m$ for $l>1$, and $C_{0}=\alpha_{i}$. Moreover, applying Lemma 5.3 with (5.1) we have the distance distortion estimate for any $x, y \in M$ with $d_{g_{i}}(x, y) \leq \varepsilon_{i}$ :

$$
\left|d_{g_{i}}(x, y)-d_{g_{i}\left(\varepsilon_{i}^{2}\right)}(x, y)\right| \leq \Psi_{D}^{\prime}\left(\alpha_{i} \mid \bar{g}\right) \varepsilon_{i} .
$$

We now rescale the metrics so that $\varepsilon_{i} \mapsto 2$, setting $h_{i}(t):=4 \varepsilon_{i}^{-2} g_{i}\left(\varepsilon_{i}^{-2} t\right)$ for $t \in\left[0, \varepsilon_{i}^{2}\right]$ and $\bar{h}_{i}:=4 \varepsilon_{i}^{-2} \bar{g}$, and estimate for all $x, y \in M$ with $d_{h_{i}(0)}(x, y) \leq 2$, that

$$
\begin{aligned}
\left|d_{\bar{h}_{i}}(x, y)-d_{h_{i}(1)}(x, y)\right| & \leq\left|d_{\bar{h}_{i}}(x, y)-d_{h_{i}(0)}(x, y)\right|+\left|d_{h_{i}(0)}(x, y)-d_{h_{i}(1)}(x, y)\right| \\
& \leq \Psi\left(\delta_{i}\right)+\Psi_{D}^{\prime}\left(\alpha_{i} \mid \bar{g}\right),
\end{aligned}
$$

which approaches 0 as $i \rightarrow \infty$.

Consequently, the we have the pointed Gromov-Hausdorff distance estimate

$$
d_{p \mathrm{GH}}\left(B_{h_{i}(1)}\left(p_{i}, 1\right), B_{\bar{h}_{i}}\left(p_{i}, 1\right)\right)<\Psi\left(\delta_{i}\right)+\Psi_{D}^{\prime}\left(\alpha_{i} \mid \bar{g}\right),
$$

and such Gromov-Hausdorff distance bounds are realized by the identity map.

Since $\left\{\varepsilon_{i}\right\} \subset[0,1]$ and $\left\{p_{i}\right\} \subset M$, by the compactness we may pass to convergent subsequences, still denoted by $\left\{\varepsilon_{i}\right\}$ and $\left\{p_{i}\right\}$, respectively. We denote $\lim _{i} \varepsilon_{i}=\varepsilon$ and $\lim _{i} p_{i}=p \in M$. There are two possibilities: either $\varepsilon_{i} \rightarrow \varepsilon>0$ or $\varepsilon=0$.

If $\varepsilon>0$, we notice that $\bar{h}_{i}=4 \varepsilon_{i}^{-2} \bar{g} \rightarrow 4 \varepsilon^{-2} \bar{g}$ smoothly as $i \rightarrow \infty$. Moreover, since $M$ is compact, the collection of geodesic balls $\left\{B_{\bar{h}_{i}}\left(p_{i}, 1\right)\right\}$ has uniformly bounded geometry - the geometry is ultimately bounded by that of $\overline{B_{4 \varepsilon^{-2} \bar{g}}(p, 2)} \subset M$. Consequently, there is a smooth limit metric $\bar{h}_{\infty}$ such that as $i \rightarrow \infty$,

$$
\left(B_{\bar{h}_{i}}\left(p_{i}, 1\right), \bar{h}_{i}\right) \stackrel{p \mathrm{CG}}{\longrightarrow}\left(B_{4 \varepsilon^{-2} \bar{g}}(p, 1), \bar{h}_{\infty}\right) .
$$

Here $p$ CG means pointed Cheeger-Gromov (smooth) convergence. On the other hand, by (5.4), it is clear that as $i \rightarrow \infty$,

$$
\left(B_{h_{i}(1)}\left(p_{i}, 1\right), h_{i}(1)\right) \stackrel{p \mathrm{GH}}{\longrightarrow}\left(B_{4 \varepsilon^{-2} \bar{g}}(p, 1), \bar{h}_{\infty}\right) .
$$

However, since the metrics $\left\{h_{i}(1)\right\}$ has uniformly controlled regularity (5.3), this last convergence must also be in the pointed Cheeger-Gromov sense. Say, the limit metric $h_{\infty}$ is defined on $B_{4 \varepsilon^{-2} \bar{g}}(p, 1)$ and we have $h_{\infty} \equiv \bar{h}_{\infty}$. But the pointed Cheeger-Gromov convergence also implies, by (5.2), that

$$
\left|h_{\infty}-\bar{h}_{\infty}\right|_{C^{k}}(p)=\lim _{i \rightarrow \infty}\left|h_{i}(1)-\bar{h}_{i}\right|_{C^{k}}\left(p_{i}\right) \geq \bar{\eta}_{1},
$$

and this contradicts the conclusion $h_{\infty} \equiv \bar{h}_{\infty}$. 
If $\varepsilon=0$, since $\bar{h}_{i}$ is nothing but the metric $\bar{g}$ scaled up, $M$ is compact and $\varepsilon_{i} \rightarrow 0$, it is clear that $B_{\bar{h}_{i}}\left(p_{i}, 1\right) \stackrel{p \mathrm{CG}}{\longrightarrow} \mathbb{B}^{m}(1)$ as $i \rightarrow \infty$. By $(5.4)$, we then have $B_{h_{i}(1)}\left(p_{i}, 1\right) \stackrel{p \mathrm{GH}}{\longrightarrow} \mathbb{B}^{m}(1)$ as $i \rightarrow \infty$. However, the uniform regularity control (5.3) improves the convergence also to pointed smooth Cheeger-Gromov convergence, i.e., as $i \rightarrow \infty$ we in fact have

$$
\left(B_{h_{i}(1)}\left(p_{i}, 1\right), h_{i}(1)\right) \stackrel{p \mathrm{CG}}{\longrightarrow}\left(\mathbb{B}^{m}(1), h_{\infty}\right),
$$

with the limit smooth metric $h_{\infty} \equiv g_{\text {Euc. }}$ But by (5.2) and (5.3) we have

$$
\left|h_{\infty}-g_{\mathrm{Euc}}\right|_{C^{k}}(o)=\lim _{i \rightarrow \infty}\left|h_{i}(1)-\bar{h}_{i}\right|_{C^{k}}\left(p_{i}\right) \geq \bar{\eta}_{1}
$$

which is a contradiction.

We now return to the proof of the theorem. Fix the $\alpha$ and $\delta=: \bar{\eta}_{2}$ determined by $\bar{g}$ through the claim, then for any smooth Riemannian metric $g$ with $\|g-\bar{g}\|_{C^{0}(M, \bar{g})}<\bar{\eta}_{2}$, we see that the evolved metric $g\left(\varepsilon^{2}\right) \in B_{C^{k}(M, \bar{g})}\left(\bar{g}, \bar{\eta}_{1}\right)$, and thus the Ricci flow continuing from $g\left(\varepsilon^{2}\right)$ exists for all time and converges to a Ricci-flat Kähler metric in $B_{C^{k}(M, \bar{g})}\left(\bar{g}, \bar{\eta}_{0}\right)$.

The proof of the first claim in Theorem 5.1 is now a simple combination of Theorems 5.2 and 2.3.

Proof of Theorem 5.1(1). By Theorem 2.3 we may find some $\bar{\eta}_{3} \in(0, \varepsilon(m))$ determined by $\bar{g}$ with $\Psi_{\mathrm{CFG}}\left(\bar{\eta}_{3} \mid m\right) \leq \frac{1}{2} \bar{\eta}_{2}$, and set $\beta(\bar{g}):=\bar{\eta}_{3}^{-1}|M|_{\bar{g}}$. Since $\max _{M}\left|\mathbf{K}_{\bar{g}}\right|_{\bar{g}} \leq 1$, if we have $\beta(\bar{g})<1$, then the original work of Cheeger, Fukaya and Gromov enables us to find an approximating metric $g^{\prime} \in B_{C^{0}(M, \bar{g})}\left(\bar{g}, \bar{\eta}_{2}\right)$ which is $(\rho, k)$-round and compatible with a nilpotent Killing structure $\mathfrak{N}$ whose orbits are of diameter less than $\bar{\eta}_{2}$. Now by Theorem 5.2 and the fact that $g^{\prime} \in B_{C^{0}(M, \bar{g})}\left(\bar{g}, \bar{\eta}_{2}\right)$, the Ricci flow starting from $g^{\prime}$ exists for all time and converges to a Ricci-flat Kähler metic $g:=g^{\prime}(\infty) \in B_{C^{k}(M, \bar{g})}\left(\bar{g}, \bar{\eta}_{0}\right)$. Since the Ricci flow is intrinsic, the infinitesimal isometries are preserved (i.e., it preserves the Killing vector fields). Consequently, $\mathfrak{N}$ is still a nilpotent Killing structure compatible with $g$, which is our desired metric.

\subsection{Reduction of the diffeomorphism type}

In this sub-section we prove the second claim of Theorem 5.1. Under the assumption that $\beta(\bar{g})<1$ and by the choice of $\bar{\eta}_{3}$, we see that $M$ admits a nilpotent Killing structure $\mathfrak{N}$. Notice that $\mathfrak{N}$ is compatible with $g$ in that the local nilpotent group actions on $M$ are isometric with respect to $g$. In fact, since $M$ is very collapsed with bounded diameter, the structure is pure, i.e., there is a single nilpotent Lie algebra $\mathfrak{n}$ such that the germ of the acting Lie group at every point has its identity component generated by $\mathfrak{n}$. Moreover, this gives us a singular Riemannian submersion $f: M \rightarrow X$ over some collapsing limit space $\left(X, d_{X}\right)$ whose topologcial structure is described by Theorem 2.2.

In fact, the nilpotent Killing structure $\mathfrak{N}$ determines a (singular) Riemannian foliation $\mathcal{N}$ (see [64]), defined by the distribution of Killing vector fields tangent to the orbits of the structure $\mathfrak{N}$. Clearly, the leaf of $\mathcal{N}$ passing through $p$ is $\mathcal{O}(p)$, the orbit of $p$ under the nilpotent Killing structure $\mathfrak{n}$, and it is also a component of the fiber $f^{-1}(f(p))$. Within $\mathcal{N}$ we can define the central distribution $\mathcal{F}$, consisting of the center $C\left(\mathcal{N}_{p}\right) \unlhd \mathcal{N}_{p}$ at every $p \in M$. This defines another (singular) Riemannian foliation by the Frobinius theorem - in fact, this defines an $F$-structure $\mathfrak{F}$ a la Cheeger and Gromov [16, 17].

The leaf space of $\mathcal{N}$ (or equivalently the orbit space of $\mathfrak{N}$ ) is isometric to the collapsing limit $\left(X, d_{X}\right)$, and the leaf space of $\mathcal{F}$ is isometric to a metric space $\left(W, d_{W}\right)$. Recalling our descriptions in Section 2.1 (according to [35, Theorem 0.5]), we have $X=\tilde{\mathcal{R}} \sqcup \tilde{\mathcal{S}}$, where $\tilde{\mathcal{R}}$ is an open (incomplete) Riemannian orbifold, and $\tilde{\mathcal{S}}$ consists of corner points, i.e., those $x \in X$ 
with $\operatorname{dim} G_{x}>0$. For such an $x, G_{x}$ has its non-trivial identity component as a torus, and more significantly, for any $p \in f^{-1}(x)$, the infinitesimal action of $G_{x}$ is contained in $\mathcal{F}_{p}=C\left(\mathcal{N}_{p}\right)$; see [35, Lemma 5.1]. Let $\mathfrak{g}_{p}$ denote the Lie algebra of $G_{x}$ for any $p \in f^{-1}(x)$, then $\mathfrak{g}_{p} \cong \mathbb{R}^{d}$ as Lie algebras, with $d=0$ when $x \in \tilde{\mathcal{R}}$ and $d \in\{1, \ldots, m-\operatorname{dim} X\}$ when $x \in \tilde{\mathcal{S}}$.

Clearly, $\mathcal{N}_{p}$, the distribution $\mathcal{N}$ located at each $p \in M$, is isomorphic to $\mathfrak{n} / \mathfrak{g}_{p}$ as Lie algebras, and $\mathcal{F}_{p}$ is isomorphic to $C(\mathfrak{n}) / \mathfrak{g}_{p}$ since $\mathfrak{g}_{p} \unlhd C(\mathfrak{n})$. Consequently, the foliation $\mathcal{N}$ is a Riemannian foliation (i.e., non-singular) if and only if $\tilde{\mathcal{S}}=\varnothing$ (which is also equivalent to saying that the nilpotent Killing structure $\mathfrak{N}$ is polarized), and the same conclusion holds for $\mathcal{F}$ and $\mathfrak{F}$. In fact, by [35, Theorem 0.5], the possibly singular Riemannian foliations $\mathcal{N}$ and $\mathcal{F}$ are always linearized; see [64]. We will denote $k^{\prime}=\operatorname{dim} C(\mathfrak{n}) \leq m-\operatorname{dim} X$.

The $\mathfrak{N}$ (and thus $\mathfrak{F}$ ) invariant metric $g$, when restricted to a leaf of $\mathcal{F}$, defines a non-negative definite 2-tensor field $G$ : for any two vector fields $\xi, \zeta \in \Gamma(\mathcal{F}, M), G(\xi, \zeta):=g(\xi, \zeta)$, which is left invariant along the leaves of $\mathcal{F}$. Consequently, the central density $-\operatorname{det} G-$ is a non-negative basic function for $\mathcal{F}$, i.e., it descends to a non-negative function on $W$; see also [51]. Clearly, $\operatorname{det} G$ is smooth around the regular leaves of $\mathcal{F}$, i.e., those leaves whose tangents are isomorphic to $C(\mathfrak{n})$. By the previous discussion on $\mathcal{F}_{p}$ for $p \in f^{-1}(\tilde{\mathcal{S}})$, we see that $\operatorname{det} G$ vanishes exactly on $f^{-1}(\tilde{\mathcal{S}})$, i.e., $\{p \in M: \operatorname{det} G(p)=0\}=f^{-1}(\tilde{\mathcal{S}})$. This is because at $p \in f^{-1}(\tilde{\mathcal{S}})$ we can extend $G$ by 0 on $\mathcal{F}_{p} \oplus \mathfrak{g}_{p} \cong C(\mathfrak{n})$; compare also [33, Theorem 0.6]. Notice that $G$ induces a left invariant Riemannian metric on the leaves of $\mathcal{F}$; but since $\mathcal{F}$ consists of commuting vector fields, $G$ is bi-invariant and actually flat along the leaves of $\mathcal{F}$.

In the formulas below, we will use the Roman letters $i, j, k, l$ to index the coordinates along the leaf directions of $\mathcal{F}$, and for directions perpendicular to a leaf of $\mathcal{F}$, we use the Greek letters $\alpha, \beta$ as indices. Moreover, we employ the Einstein summation convention, adding the repeated indices. As the basic function $\operatorname{det} G$ is constant along the leaves of $\mathcal{F}$, if it were not a constant throughout $M$, then $\max _{M} \ln \operatorname{det} G=\max _{W} \ln \operatorname{det} G$ is attained at some $p \in f^{-1}(\tilde{\mathcal{R}})$, since $\operatorname{det} G \geq 0$ and $\left.\operatorname{det} G\right|_{f^{-1}(\tilde{\mathcal{S}})} \equiv 0$. By the O'Neill's formula [67] applied to the (singular) Riemannian foliation $\mathcal{F}$ in a small enough open neighborhood around $p \in M$ and the flatness of the leaves, we have some locally defined basic 1-form $A_{\alpha \beta}^{l}$ such that

$$
\mathbf{R c}_{i j}=-\frac{1}{2}\left(G_{i j ; \alpha \alpha}-G_{i k, \alpha} G_{j k, \alpha}\right)-\frac{1}{4} G^{k l} G_{k l, \alpha} G_{i j, \alpha}+\frac{1}{4} G_{i k} G_{j l} A_{\alpha \beta}^{k} A_{\alpha \beta}^{l} .
$$

Now tracing by $G^{i j}$ on the leaf directions and by the Ricci-flatness of the metric on $M$, we see that

$$
\frac{1}{2} \Delta^{\perp} \ln \operatorname{det} G+\frac{1}{4}\left|\nabla^{\perp} \ln \operatorname{det} G\right|^{2}=|A|^{2} .
$$

Here $\nabla^{\perp}$ and $\Delta^{\perp}$ denote the derivatives taken perpendicular to the leaf directions. However, since $\ln \operatorname{det} G(p)=\max _{M} \ln \operatorname{det} G$, we must have $\Delta^{\perp} \ln \operatorname{det} G(p)<0$ and $\nabla^{\perp} \ln \operatorname{det} G(p)=0-$ this will contradict the non-negativity of the right-hand side of (5.6). Consequently, we know that $\operatorname{det} G$ is a positive constant. This implies that $\tilde{\mathcal{S}}=\varnothing$, i.e., $X=\tilde{\mathcal{R}}$ is a Riemannian orbifold. Moreover, $\mathcal{F}$ is a Riemannian foliation - the orbit space $W$ of $\mathcal{F}$ is also a Reimannian orbifold.

Clearly, $G_{i j}$ descends to germs of smooth functions on the regular part of $W$; if $w \in W$ is a singularity, it can only be an orbifold point and we may pull the corresponding quantities back to its local orbifold covering - the differentials of $G_{i j}$ are well-defined throughout $W$. Notice that $\nabla_{W} G_{i j}=\nabla^{\perp} G_{i j}$ and $\Delta_{W} G_{i j}=\Delta^{\perp} G_{i j}$. Moreover, by (5.6) the constancy of $\ln \operatorname{det} G$ ensures that $|A| \equiv 0$ throughout $X$, and thus by (5.5),

$$
\Delta_{W} G_{i j}=\Delta^{\perp} G_{i j}=G_{i k, \alpha} G_{j k, \alpha} .
$$

If we further check the O'Neill's formula for the regular part of $W$, the vanishing of $\left|\nabla^{\perp} \ln \operatorname{det} G\right|$ and $|A|$, together with the Ricci-flatness of $M$ tell that

$$
\left(\mathbf{R c}_{W}\right)_{\alpha \beta}=\frac{1}{4} G_{i j, \alpha} G_{i j, \beta} .
$$


We notice that the quantity $\left|\nabla_{W} G\right|^{2}$ is a globally defined non-negative smooth function on $W$. By the compactness of $W$, if $\left|\nabla_{W} G\right|^{2} \not \equiv 0$, then we have $\Delta_{W}\left|\nabla_{W} G\right|^{2}(w)<0$ at the maximum point $w \in W$. On the other hand, since $\operatorname{det} G$ is a constant, we may view the $\left[G_{i j}\right]$ as a matrix valued map $G: U \rightarrow \mathrm{SL}\left(k^{\prime}, \mathbb{R}\right) / \mathrm{SO}\left(k^{\prime}\right)$, with $U \subset W$ being an open neighborhood of $w$ where we can write down $G$ in coordinates. We now notice that the codomain $S_{k^{\prime}}:=\operatorname{SL}\left(k^{\prime}, \mathbb{R}\right) / \mathrm{SO}\left(k^{\prime}\right)$ is a negatively curved symmetric space. Now by the Bochner formula, we can calculate at $w \in U$ to see

$$
\begin{aligned}
\Delta_{W}\left|\nabla_{W} G\right|^{2}(w) & =2\left|\nabla_{W} \nabla_{W} G\right|^{2}(w)+\left(\mathbf{R c}_{W}\right)_{\alpha \beta} G_{i j, \alpha} G_{i j, \beta}(w)-\left(G^{*} \mathbf{R}_{S_{k^{\prime}}}\right)_{\alpha \beta \beta \alpha}(w) \\
& >\frac{1}{4}\left|\nabla_{W} G\right|^{4}(w)>0,
\end{aligned}
$$

which is impossible. Therefore, we see the tensor $\nabla^{\perp} G \equiv 0$ on $M$. Consequently, we see that (5.7) reduces to $\mathbf{R c}_{W} \equiv 0$. Moreover, as $\nabla^{\perp} G$ stands for the second fundamental form of the leaves of $\mathcal{F}$, its vanishing tells that the the leaves of $\mathcal{F}$ are totally geodesic. So the Riemannian metric $g$ locally splits, and by the left invariance of $g$ with respect to $\mathfrak{N}$, this implies the splitting of the nilpotent Killing structure, i.e., passing through each point $p \in M$, we have the splitting of Lie algebras $\mathcal{N}_{p}=\mathcal{F}_{p} \oplus \mathcal{N}_{p}^{\prime}$ for some nilpotent Lie algebra $\mathcal{N}_{p}^{\prime}$. This however leads to $\mathcal{F}=\mathcal{N}$ : otherwise, $\mathcal{N}_{p}^{\prime} \neq 0$ at some $p \in M$, and by the nilpotency it has to have a non-trivial center $C\left(\mathcal{N}_{p}^{\prime}\right)$, but the above splitting shows that $\mathcal{F}_{p}=C\left(\mathcal{N}_{p}\right)=\mathcal{F}_{p} \oplus C\left(\mathcal{N}_{p}^{\prime}\right)$, which is absurd. Consequently, we have $\mathcal{F}=\mathcal{N}, X \equiv W$ as Ricci-flat orbifolds, and that $M$ fibers over $X$ by flat tori with totally geodesic fibers (the leaves of $\mathcal{N}$ ).

\section{Acknowledgements}

The second author was partially supported by NSFC Grant 11821101, Beijing Natural Science Foundation Z19003, and a research fund from Capital Normal University. The third author is partially supported by the General Program of the National Natural Science Foundation of China (Grant No. 11971452) and a research fund of USTC. The authors would like to thank anonymous referees for their careful proofreading and helpful comments of the paper.

\section{References}

[1] Bamler R.H., Zhang Q.S., Heat kernel and curvature bounds in Ricci flows with bounded scalar curvature, Adv. Math. 319 (2017), 396-450, arXiv:1501.01291.

[2] Bamler R.H., Zhang Q.S., Heat kernel and curvature bounds in Ricci flows with bounded scalar curvature Part II, Calc. Var. Partial Differential Equations 58 (2019), 49, 14 pages, arXiv:1506.03154.

[3] Bochner S., Vector fields and Ricci curvature, Bull. Amer. Math. Soc. 52 (1946), 776-797.

[4] Buser P., Karcher H., Gromov's almost flat manifolds, Astérisque 81 (1981), 148 pages.

[5] Buyalo S.V., Collapsing manifolds of nonpositive curvature. I, Leningrad Math. J. 1 (1990), 1135-1155.

[6] Buyalo S.V., Collapsing manifolds of nonpositive curvature. II, Leningrad Math. J. 1 (1990), 1371-1399.

[7] Cai Q., Rong X., Collapsing construction with nilpotent structures, Geom. Funct. Anal. 18 (2009), 15031524.

[8] Cao J., Cheeger J., Rong X., Splittings and Cr-structures for manifolds with nonpositive sectional curvature, Invent. Math. 144 (2001), 139-167.

[9] Cao J., Cheeger J., Rong X., Local splitting structures on nonpositively curved manifolds and semirigidity in dimension 3, Comm. Anal. Geom. 12 (2004), 389-415.

[10] Cavalletti F., Mondino A., Sharp and rigid isoperimetric inequalities in metric-measure spaces with lower Ricci curvature bounds, Invent. Math. 208 (2017), 803-849, arXiv:1502.06465.

[11] Chau A., Tam L.-F., Yu C., Pseudolocality for the Ricci flow and applications, Canad. J. Math. 63 (2011), 55-85, arXiv:math.DG/0701153. 
[12] Cheeger J., Finiteness theorems for Riemannian manifolds, Amer. J. Math. 92 (1970), 61-74.

[13] Cheeger J., Colding T.H., On the structure of spaces with Ricci curvature bounded below. I, J. Differential Geom. 46 (1997), 406-480.

[14] Cheeger J., Fukaya K., Gromov M., Nilpotent structures and invariant metrics on collapsed manifolds, J. Amer. Math. Soc. 5 (1992), 327-372.

[15] Cheeger J., Gromoll D., The splitting theorem for manifolds of nonnegative Ricci curvature, J. Differential Geometry 6 (1971), 119-128.

[16] Cheeger J., Gromov M., Collapsing Riemannian manifolds while keeping their curvature bounded. I, J. Differential Geom. 23 (1986), 309-346.

[17] Cheeger J., Gromov M., Collapsing Riemannian manifolds while keeping their curvature bounded. II, J. Differential Geom. 32 (1990), 269-298.

[18] Cheeger J., Jiang W., Naber A., Rectifiability of singular sets in noncollapsed spaces with Ricci curvature bounded below, arXiv:1805.07988.

[19] Cheeger J., Rong X., Collapsed Riemannian manifolds with bounded diameter and bounded covering geometry, Geom. Funct. Anal. 5 (1995), 141-163.

[20] Cheeger J., Rong X., Existence of polarized $F$-structures on collapsed manifolds with bounded curvature and diameter, Geom. Funct. Anal. 6 (1996), 411-429.

[21] Chen L., Rong X., Xu S., Quantitative volume space form rigidity under lower Ricci curvature bound II, Trans. Amer. Math. Soc. 370 (2018), 4509-4523, arXiv:1606.05709.

[22] Chen L., Rong X., Xu S., Quantitative volume space form rigidity under lower Ricci curvature bound I, J. Differential Geom. 113 (2019), 227-272, arXiv:1604.06986.

[23] Chen X., Wang B., Space of Ricci flows I, Comm. Pure Appl. Math. 65 (2012), 1399-1457, arXiv:0902.1545.

[24] Chen X., Wang B., Remarks of weak-compactness along Kähler Ricci flow, in Proceedings of the Seventh International Congress of Chinese Mathematicians, Vol. II, Adv. Lect. Math. (ALM), Vol. 44, Int. Press, Somerville, MA, 2019, 203-233, arXiv:1605.01374.

[25] Chen X., Wang B., Space of Ricci flows (II) - Part B: Weak compactness of the flows, J. Differential Geom. 116 (2020), 1-123, arXiv:1405.6797.

[26] Colding T.H., Shape of manifolds with positive Ricci curvature, Invent. Math. 124 (1996), $175-191$.

[27] Colding T.H., Ricci curvature and volume convergence, Ann. of Math. 145 (1997), 477-501.

[28] Colding T.H., Naber A., Sharp Hölder continuity of tangent cones for spaces with a lower Ricci curvature bound and applications, Ann. of Math. 176 (2012), 1173-1229, arXiv:1102.5003.

[29] Dai X., Wang X., Wei G., On the variational stability of Kähler-Einstein metrics, Comm. Anal. Geom. 15 (2007), 669-693.

[30] Dai X., Wei G., Ye R., Smoothing Riemannian metrics with Ricci curvature bounds, Manuscripta Math. 90 (1996), 49-61, arXiv:dg-ga/9411014.

[31] Fang F., Rong X., Positive pinching, volume and second Betti number, Geom. Funct. Anal. 9 (1999), 641-674.

[32] Fang F., Rong X., The second twisted Betti number and the convergence of collapsing Riemannian manifolds, Invent. Math. 150 (2002), 61-109.

[33] Fukaya K., Collapsing of Riemannian manifolds and eigenvalues of Laplace operator, Invent. Math. 87 (1987), 517-547.

[34] Fukaya K., Collapsing Riemannian manifolds to ones of lower dimensions, J. Differential Geom. 25 (1987), 139-156.

[35] Fukaya K., A boundary of the set of the Riemannian manifolds with bounded curvatures and diameters, J. Differential Geom. 28 (1988), 1-21.

[36] Fukaya K., Collapsing Riemannian manifolds to ones with lower dimension. II, J. Math. Soc. Japan 41 (1989), 333-356.

[37] Fukaya K., Metric Riemannian geometry, in Handbook of Differential Geometry, Vol. II, Elsevier/NorthHolland, Amsterdam, 2006, 189-313.

[38] Greene R.E., Wu H., Lipschitz convergence of Riemannian manifolds, Pacific J. Math. 131 (1988), $119-141$.

[39] Gromov M., Almost flat manifolds, J. Differential Geometry 13 (1978), 231-241. 
[40] Gromov M., Structures métriques pour les variétés riemanniennes, Textes Mathématiques, Vol. 1, CEDIC, Paris, 1981.

[41] Gross M., Tosatti V., Zhang Y., Collapsing of abelian fibered Calabi-Yau manifolds, Duke Math. J. 162 (2013), 517-551.

[42] Gross M., Tosatti V., Zhang Y., Gromov-Hausdorff collapsing of Calabi-Yau manifolds, Comm. Anal. Geom. 24 (2016), 93-113, arXiv:1304.1820.

[43] Gross M., Wilson P.M.H., Large complex structure limits of K3 surfaces, J. Differential Geom. 55 (2000), 475-546, arXiv:math.DG/0008018.

[44] Hamilton R.S., Three-manifolds with positive Ricci curvature, J. Differential Geometry 17 (1982), 255-306.

[45] Hamilton R.S., The formation of singularities in the Ricci flow, in Surveys in Differential Geometry, Vol. II (Cambridge, MA, 1993), Int. Press, Cambridge, MA, 1995, 7-136.

[46] Hein H.-J., Sun S., Viaclovsky J., Zhang R., Nilpotent structures and collapsing Ricci-flat metrics on $K 3$ surfaces, arXiv:1807.09367.

[47] Hochard R., Short-time existence of the Ricci flow on complete, non-collapsed 3-manifolds with Ricci curvature bounded from below, arXiv:1603.08726.

[48] Huang H., Fibrations and stability for compact group actions on manifolds with local bounded Ricci covering geometry, Front. Math. China 15 (2020), 69-89, arXiv:2002.07383.

[49] Huang H., Kong L., Rong X., Xu S., Collapsed manifolds with Ricci bounded covering geometry, Trans. Amer. Math. Soc. 373 (2020), 8039-8057, arXiv:1808.03774.

[50] Huang H., Rong X., Collapsed manifolds with Ricci curvature and local rewinding volume bounded below, in preparation.

[51] Huang S., On the long-time behavior of immortal Ricci flows, arXiv:1908.05410.

[52] Huang S., Notes on Ricci flows with collapsing initial data (I): Distance distortion, Trans. Amer. Math. Soc. 373 (2020), 4389-4414, arXiv:1808.07394.

[53] Huang S., Wang B., Rigidity of the first Betti number via Ricci flow smoothing, arXiv:2004.09762.

[54] Huang S., Wang B., Ricci flow smoothing for locally collapsing manifolds, arXiv:2008.09956.

[55] Kapovitch V., Mixed curvature almost flat manifolds, arXiv:1911.09212.

[56] Kapovitch V., Li N., On dimensions of tangent cones in limit spaces with lower Ricci curvature bounds, J. Reine Angew. Math. 742 (2018), 263-280, arXiv:1506.02949.

[57] Kapovitch V., Wilking B., Structure of fundamental groups of manifolds with Ricci curvature bounded below, arXiv:1105.5955.

[58] Kontsevich M., Soibelman Y., Homological mirror symmetry and torus fibrations, in Symplectic Geometry and Mirror Symmetry (Seoul, 2000), World Sci. Publ., River Edge, NJ, 2001, 203-263, arXiv:math.SG/0011041.

[59] Ledrappier F., Wang X., An integral formula for the volume entropy with applications to rigidity, J. Differential Geom. 85 (2010), 461-477, arXiv:0911.0370.

[60] Li Y., SYZ conjecture for Calabi-Yau hypersurfaces in the Fermat family, arXiv:1912.02360.

[61] Lott J., Some geometric properties of the Bakry-Émery-Ricci tensor, Comment. Math. Helv. 78 (2003), 865-883, arXiv:math.DG/0211065.

[62] Lott J., Dimensional reduction and the long-time behavior of Ricci flow, Comment. Math. Helv. 85 (2010), 485-534, arXiv:0711.4063.

[63] Lu P., A local curvature bound in Ricci flow, Geom. Topol. 14 (2010), 1095-1110, arXiv:0906.3784.

[64] Molino P., Riemannian foliations, Progress in Mathematics, Vol. 73, Birkhäuser Boston, Inc., Boston, MA, 1988.

[65] Naber A., Tian G., Geometric structures of collapsing Riemannian manifolds II, J. Reine Angew. Math. 744 (2018), 103-132, arXiv:0804.2275.

[66] Naber A., Zhang R., Topology and $\varepsilon$-regularity theorems on collapsed manifolds with Ricci curvature bounds, Geom. Topol. 20 (2016), 2575-2664, arXiv:1412.1326.

[67] O'Neill B., The fundamental equations of a submersion, Michigan Math. J. 13 (1966), 459-469.

[68] Perelman G., The entropy formula for the Ricci flow and its applications, arXiv:math.DG/0211159. 
[69] Petersen P., Wei G., Ye R., Controlled geometry via smoothing, Comment. Math. Helv. 74 (1999), 345-363, arXiv:dg-ga/9508012.

[70] Petrunin A., Rong X., Tuschmann W., Collapsing vs. positive pinching, Geom. Funct. Anal. 9 (1999), 699-735.

[71] Petrunin A., Tuschmann W., Diffeomorphism finiteness, positive pinching, and second homotopy, Geom. Funct. Anal. 9 (1999), 736-774.

[72] Rong X., The existence of polarized F-structures on volume collapsed 4-manifolds, Geom. Funct. Anal. 3 (1993), 474-501.

[73] Rong X., The limiting eta invariants of collapsed three-manifolds, J. Differential Geom. 37 (1993), 535-568.

[74] Rong X., Rationality of geometric signatures of complete 4-manifolds, Invent. Math. 120 (1995), 513-554.

[75] Rong X., Collapsed manifolds with bounded sectional curvature and applications, in Surveys in Differential Geometry, Vol. XI, Surv. Differ. Geom., Vol. 11, Int. Press, Somerville, MA, 2007, 1-23.

[76] Rong X., Manifolds of Ricci curvature and local rewinding volume bounded below, Sci. Sin. Math. 48 (2018), 791-806.

[77] Rong X., A new proof of Gromov's theorem on almost, arXiv:1906.03377.

[78] Rong X., A generalized Gromov's theorem on almost flat manifolds and applications, in preparation.

[79] Ruh E.A., Almost flat manifolds, J. Differential Geometry 17 (1982), 1-14.

[80] Shi W.-X., Deforming the metric on complete Riemannian manifolds, J. Differential Geom. 30 (1989), 223-301.

[81] Strominger A., Yau S.-T., Zaslow E., Mirror symmetry is T-duality, Nuclear Phys. B 479 (1996), 243-259, arXiv:hep-th/9606040.

[82] Tian G., Wang B., On the structure of almost Einstein manifolds, J. Amer. Math. Soc. 28 (2015), 1169-1209, arXiv:1202.2912.

[83] Tosatti V., Collapsing Calabi-Yau manifolds, Surv. Differ. Geom. 23 (2020), 305-337, arXiv:2003.00673.

[84] Wang B., The local entropy along Ricci flow Part A: the no-local-collapsing theorems, Camb. J. Math. 6 (2018), 267-346, arXiv:1706.08485.

[85] Yano K., Bochner S., Curvature and Betti numbers, Annals of Mathematics Studies, Vol. 32, Princeton University Press, Princeton, N.J., 1953. 